\title{
Shock Propagation in Polydisperse Bubbly Liquids
}

Keita Ando, Tim Colonius, and Christopher E. Brennen

\begin{abstract}
We investigate the shock dynamics of liquid flows containing small gas bubbles, based on a continuum bubbly flow model. Particular attention is devoted to the effects on shock dynamics of distributed bubble sizes and gas-phase nonlinearity. Ensemble-averaged conservation laws for polydisperse bubbly flows, together with a Rayleigh-Plesset-type model for single bubble dynamics, form the starting point for these studies. Numerical simulations of one-dimensional shock propagation reveal that phase cancellations in oscillations of different-sized bubbles can lead to an apparent damping of the averaged shock dynamics. Experimentally we study the propagation of finite-amplitude waves in a bubbly liquid in a de-formable tube. The ensemble-averaged bubbly flow model is extended to quasi-one-dimensional cases and the corresponding steady shock relations are derived. These account for the compressibilities associated with the tube deformation as well as the bubbles and host liquid. A comparison between the theory and the water-hammer experiment suggests that the gas-phase nonlinearity plays an essential role in the propagation of strong shocks.
\end{abstract}

\section{Introduction}

A number of complex phenomena in bubbly liquids can be attributed to the interaction of the flow and the dynamics of dispersed bubbles. Even for the case of dilute flows (bubble volume fraction less than $1 \%$ ), the flow structure can be significantly altered by bubble dynamics. The presence of compressible bubbles in a stiff liquid means that the effective compressibility of

Keita Ando Keio University, e-mail: kando@mech.keio.ac.jp

Tim Colonius California Institute of Technology, e-mail: colonius@caltech.edu

Christopher E. Brennen California Institute of Technology, e-mail: brennen@caltech.edu 
the mixture can deviate drastically from the liquid compressibility. If bubbles respond in phase with the flow excitation, the mixture becomes less stiff and the resulting wave speed is reduced. Since the bubble response depends on the excitation frequency (as well as amplitude), the mixture compressibility and the corresponding phase velocity are regulated by the length scale of the waves. That is, waves are dispersive due to bubble dynamics. Nonlinearity associated with gas-phase compressibility is another issue, particularly evident in strong shocks.

In the modeling and simulation of the dynamics of dispersed bubbly flows, it has been a challenge to resolve wave dispersion and nonlinear bubble dynamics. Direct numerical simulations of wave interaction with individual bubbles $[27,28,53,70]$ can shed light on the detailed flow structures but are impractical for large-scale flow computations. For practical applications, for example in hydraulic machinery [5, 14], underwater explosions [22,46], or shockwave lithotripsy [6, 49], we favor simulating mixture-averaged dynamics (rather than solutions to individual realizations) based on continuum models in which the bubbly mixture is treated as a continuum. Averaging approaches allow us to remove stiffness resulting from individual bubble dynamics and thus reduce the computational effort. In such an approach, a choice of the constitutive relation for a model closure is required. The simplest choice is a barotropic relation $[15,17]$ that ignores the bubble dynamics and is therefore incapable of capturing wave dispersion. Alternatively, to account for the unsteadiness associated with the bubbles, a Rayleigh-Plesset-type model for the single bubble dynamics can be coupled with mixture-averaged conservation laws $[25,57,86]$. However, to further reduce the computational effort, monodisperse bubbles (of the same size) are often assumed.

We consider the canonical example of shock propagation through a bubbly liquid in a tube. In the pioneering work of Campbell and Pitcher [20] and the subsequent experiments (see for example [11, 44, 45, 60, 79]), dispersed bubbly flows were created in a vertical tube attached to a shock tube in order to generate shock propagation. Shocks in monodisperse bubbly flows exhibit an oscillatory structure that can also be numerically predicted using continuum models coupled with single-bubble-dynamic equations [44, 45, 58, 70, 78, 87]. In particular, the work of Kameda et al. [45] reveals that the shock structure in the monodisperse case is sensitive to thermal dissipation in the bubble oscillations. However, experimental observations on shocks in the polydisperse case are rather limited.

The above-mentioned experiments were confined to the case of weak shocks. Further increase in the shock strength results in bubble fission [16] and tube deformation [71]. Dynamic loading of a fluid-filled deformable tube is a classic example used to study fluid-structure interactions in water-hammer events [89]. For tubes filled with liquids (without bubbles), a linear wave speed is predicted by the Korteweg-Joukowsky model that accounts for the compressibility associated with the liquid and the structure [43, 48]. This was later extended to bubbly liquid cases [47]. On the experimental side, an underwater shock simulator has been developed to study coupled stress waves propagating in the axial direction of a fluid-filled tube $[26,31$, 40]. However, the study of finite-amplitude waves in a mixture-filled, deformable tube had not been previously reported. 
The aim of this chapter is to review recent progress in the modeling and simulation of shock dynamics of dispersed bubbly flows. In particular, we focus on the effects of polydispersity [3] and gas-phase nonlinearity

[4] on the mixture-averaged shock dynamics. In Sect. 2, the ensemble-averaged bubbly flow model (together with single-bubble-dynamic equations) is introduced and the acoustic properties of polydisperse bubbly liquids are then examined. In Sect. 3, the numerical method for shock computations is presented and one-dimensional shock propagation in a polydisperse bubbly liquid is simulated to quantify the effect of polydispersity on averaged shock dynamics. To examine the effect of gas-phase nonlinearity as well as fluid-structure interaction (FSI), shock propagation through a bubbly liquid in a deformable tube is considered in Sect. 4. The ensemble-averaged model is extended to quasi-one-dimensional configurations in order to incorporate FSI; the corresponding steady shock relations are compared to water-hammer experiments. Finally, we summarize the our findings in Sect. 5.

\section{Modeling of Continuum Bubbly Flows}

\subsection{Mixture-Averaged Conservation Laws}

In deriving a continuum model for bubbly flows, there is a need to select either time, volume, or ensemble averaging $[41,86,91]$ in order to define mixture-averaged quantities. If the system is ergodic $[8,12]$, averaged values do not depend on a choice of the averaging manner under appropriate scale separation $[3,56,65]$. Here, we use the emsemble-averaging technique of Zhang and Prosperetti [90, 91, 92] to formally derive mixture-averaged conservation laws. To be specific, physical quantities are statistially averaged over a large number of realizations of spherical bubbles in the flow; any scattering effects in an individual realization are discarded but the statistically averaged dynamics are explored. For dilute flows, direct interactions between neighboring bubbles are often ignored. In this case, the system is two-way-coupled in a sense that bubble interactions are captured indirectly through the averaged field. An attempt to account for direct bubble/bubble interactions, which is useful in particular for high void fraction flows (see for example $[13,19,35,70]$ ), is beyond our scope.

For the two-way-coupled case, we write the emsemble-averaged conservation equations as 
$\partial \rho$

$\partial \rho \mathbf{u}$

$+\nabla \cdot(\mathbf{p u u})+\nabla\left(p l-p^{\sim}\right)=\mathbf{0}$,

(2)

$\partial t \partial \alpha R^{2} R^{*}$

$$
+\nabla \cdot(\mathbf{\alpha u})=3 \alpha
$$

where $\rho$ is the mixture density, $\mathbf{u}$ is the mixture velocity, $p l$ is the averaged liquid pressure, $\alpha$ is the volume fraction of bubbles (i.e., void fraction), and $R$ and $R$ are the bubble radius and the bubble wall velocity, respectively. Here, the mixture density is approximated by $\rho=(1-\alpha) \rho l$ where $\rho l$ is the liquid density. It is assumed that the bubbles follow the ambient liquid motion (i.e., no-slip), for relative motion between the phases plays a minor role in shock propagation $[44,70]$. The liquid-phase pressure is given by the Tait equation of state [81] that assumes isentropic processes in the liquid phase:

()$_{m}$

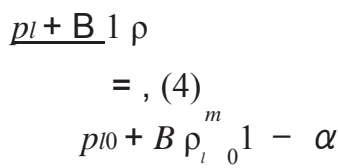

where $\rho l 0$ is the reference liquid density at the undisturbed pressure $p l 0$, and $m$ and $B$ denote the liquid stiffness and tensile strength, respectively. For water, we use $m=$ 7.15 and then determine the value of $B$, specifying the speed of sound in the liquid at a given temperature.

The phase interaction term $p^{\sim}$ in the momentum equation (2), which does not appear in classic volume-averaged equations formulated with heuristic reasoning $[85,86]$, is

$$
p^{\sim}=\alpha p l--\underline{R}_{, p_{b w}} R_{3} R_{2}{ }_{2}
$$

where $p_{b w}$ is the bubbl wall pressure in the liquid phase [15]:

$4 \mu I R^{*} 2 S$

$$
p_{b w}=p_{b}+-.(6)
$$

$R R$

Here, $p b$ is the internal bubble pressure, $\mu l$ is the liquid viscosity, and $S$ is the surface 
tension. The overbar in (5) as well as (3) denotes moments with respect to the distribution of equilibrium bubble radius $R 0$ at $p 10$. With no-slip and monodisperse assumptions, the phase interaction term (5) reduces to that of Biesheuvel and van Wijngaarden [12] or Zhang and Prosperetti [91].

To account for the effect of polydispersity, we need to evaluate the moments in (3) and (5):

$$
\phi^{-}(\mathbf{x}, t)=\stackrel{\mid \infty}{\phi\left(\mathbf{x}, t ; R_{0}\right)} f\left(R_{0}\right) \mathrm{d} R_{0},(7)
$$

0 where $\phi$ represents particle quantities (such as $R^{3}$ ) and the (normalized) bubble size distribution, $f(R 0)$, is assumed spatially uniform. The equilibrium bubble radius, $R 0$, is assumed unchanged within fluid-dynamic time scales, which are typically 


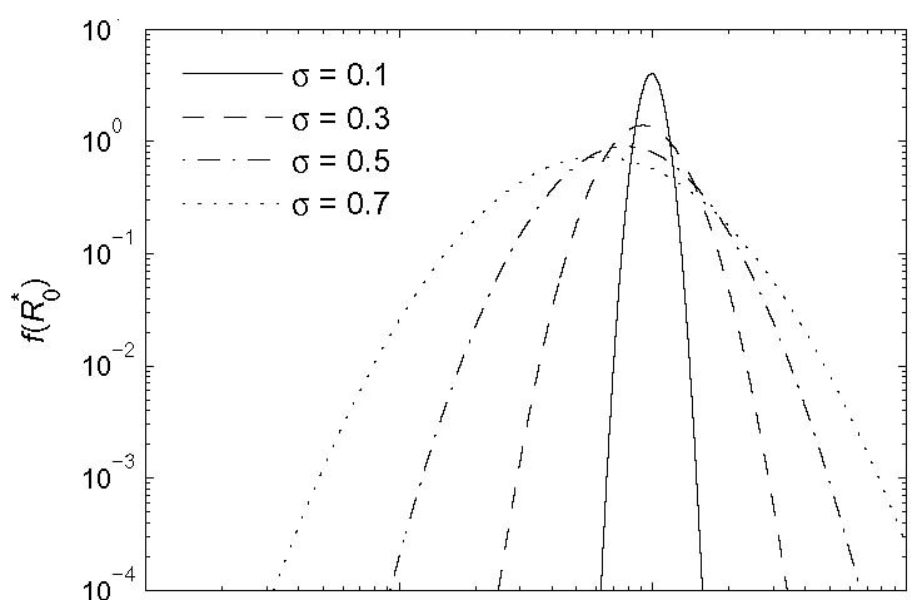

$-2-101$

10101010

$R$

0

Fig. 1 Lognormal distributions (8) with varying the standard deviation $\sigma$. The distributions are normalized in order to satisfy ${ }^{\infty} f\left(R_{0}^{*}\right) \mathrm{d} R^{*}=1$.

much shorter than those associated with mass diffusion of dissolved gases (such as air in water) $[30,62]$. As an illustrative example, we model the disribution using a lognormal function (Fig. 1) with the probable size $R^{\text {ref }}$ and standard deviation $\sigma$ :

$1 \ln ^{2} R^{*}$ 


$$
\begin{array}{r}
f\left(R_{0}^{*}\right)=\sqrt{ } \exp -,(8) \\
2 \pi \sigma R^{*}{ }_{0} 2 \sigma^{2} \\
=R_{0} / R
\end{array}
$$

where $R^{*}$. In the limit of $\sigma \rightarrow 0$, the distribution (8) reduces to the Dirac

$$
0
$$

delta function that models monodisperse mixtures. The measured distribution of gas bubble nuclei in water tunnels or seawater is reasonably fit by the lognormal distribution with $\sigma \approx 0.7[3,24]$. For example, the void fraction is computed as

$$
4 \pi
$$

$$
\alpha=n R^{3},(9)
$$

3

where $n$ is the number of bubbles per unit volume of the mixture. Provided that fission and coalescence of the bubbles do not occur, the bubble number density is conserved in time; namely

$$
\begin{aligned}
& \partial n \\
& \\
&
\end{aligned} t^{+}+\nabla \cdot(n \mathbf{u})=0 .(10)
$$

However, for strong shocks, there will arise bubble fission possibly due to a RayleighTaylor-type shape instability or a re-entrant jet $[16,29,42,68]$, so that the bubble number conservation no longer holds. 


\subsection{Single-Bubble-Dynamic Equations}

A closure of the mixture-averaged equations (1) to (3) requires evaluating individual bubble dynamics. Taking into account acoustic damping (to first order) on bubble oscillations, we use the Gilmore equation [36] that is an improvement to the incompressible Rayleigh-Plesset equation [61, 69]:

$$
()()()
$$

$$
\begin{gathered}
R^{\cdot} 3 R^{\cdot} R^{\cdot} R H^{*} R^{\cdot} \\
R R^{*} 1-\quad+R^{.^{2}} 1-=H 1++1-,(11) \\
C 23 C \text { CCC }
\end{gathered}
$$

where the dot denotes the substantial time derivative, and $H$ and $C$ are the enthalpy and the speed of sound in the liquid, respectively, at the bubble wall:

$$
\begin{aligned}
& \text { I' } \\
& { }_{p b w} \mathrm{~d} p \mathrm{~d} p_{l} \quad H=\stackrel{l}{ }, C=.(12) \\
& \rho l(p) \mathrm{d} \rho l \\
& { }^{p} \text { l }{ }^{\prime} p_{b w}
\end{aligned}
$$

In calculating $H$, the term $p l$ is taken from the averaged liquid pressure in the continuum model. Note that the averaged liquid pressure can be regarded, in the dilute limit, as far-field pressure from the bubble $[18,77]$.

Finally, the internal bubble pressure $p_{b}$ in (6) needs to be determined. The simplest choice is the polytropic gas law:

$$
\begin{aligned}
& )_{-3 \kappa} \\
& \text { R } \\
& p_{b}=p_{v}\left(T_{w}\right)+p_{g 0},(13)
\end{aligned}
$$

where $p_{v}$ is the vapor pressure at bubble wall temperature $T_{w}, p_{g 0}$ is the partial pressure of the noncondensible gas in the equilibrium state, and $\kappa$ is the polydropic index that ranges from 1 (isothermal) to the ratio of specific heats $\gamma_{g}$ (adiabatic). Here, it is assumed that the bubble contents have spatially uniform pressure. This homobaric assumption is valid since the inertia of the bubble contents is neglibible compared to the liquid inertia [66]. However, for nonlinear computations, using

(13) often gives rise to innacurate evaluations of the thermal behabior of the bubble. For resolving the thermal effect on the dynamics of bubbles, the full conservation equations for both liquid and gas phases need to be solved. If the bubble contents are perfect, the bubble energy equation can be simplified to $[59,66]$, 
$p^{\prime} b-{ }^{\prime} m k b w(14)$

$=R p b+\mathrm{R}_{v} T_{w}+$,

$R \gamma_{b} \partial r$

where $\gamma_{b}$ is the specific-heat ratio of the bubble contents, $R_{v}$ is the gas constant for

the vapor, $m$ 'is the mass flux of the vapor at the bubble wall, and kbw is the ther

mal conductivity of the bubble contents. The last term in the parenthesis stands for heat conduction, across the bubble wall, driven by the temperature gradient $(\partial T / \partial r)$ in the gas phase. Provided that the liquid is cold (well below the boiling point) and phase change occurs instantaneously, the vapor pressure can be assumed unchanged, except near the the end of a violent bubble collapse [34], with constant wall temperature. In this case, the liquid-phase thermodynamics becomes irrelevant. In the simulations of shocks in monodisperse bubbly flows [44, 45, 87], the full conservation equations for the bubble contents are solved to accurately evaluate the heat and mass fluxes in (14). However, computations of the full partial differential equations are still expensive for polydisperse cases. To further reduce the conputational effort, one may use the reduced-order model of Preston, Colonius, and Brennen [63] which constitutes a set of coupled ordinary differential equations describing diffusive effects on the single bubble dynamics. 
We now write the bubble-dynamic equations in conservation form, which is suitable for shock computations [50]. For example, the evolution of the bubble radius can be described by

$$
\begin{aligned}
& \partial n R \\
& \quad+\nabla \cdot(n R \mathbf{u})=n R \cdot,(15)
\end{aligned}
$$

$\partial t$ where the bubble radius is defined as Eulerian variable $R(\mathbf{x}, t ; R 0)$, enen though it is attributed to Lagrangian particles. Now that a large number of bubbles are assumed to exist for the mixture to be a continuum, the bubbles may be considered to be distributed continuously in space [87]. The evolution of other bubble-dynamic variables (such as $R^{\circ}$ and $p b$ ) can be written in the same fashion.

\subsection{Acoustics of Polydisperse Bubbly Liquids}

Before proceeding to shock computations in Sect. 3, we examine the effect of polydispersity on linear waves in a dilute bubbly liquid. By linearizing the continuum bubbly flow equations (Sect. 2.1) coupled with the single-bubble-dynamic model (Sect. 2.2), one may derive the dispersion relation [25], which is indentical to that from multiple scattering theory $[21,33]$ :

$$
\begin{aligned}
& \quad 11^{\infty} R 0 f(R 0) \mathrm{d} R 0 \\
& =+4 \pi n,(16) \\
& { }_{c}^{22{ }_{\omega}^{2}} \\
& m C_{l} 0_{N}-\omega^{2}+i 2 \beta \omega
\end{aligned}
$$

where $\mathrm{Cm}$ is the complex speed of sound in the mixture, $\mathrm{Cl}$ is the sonic speed in the liquid alone, $\beta$ is the bubble-dynamic damping constant, $\omega$ is the angular frequency ( $\omega$ $=2 \pi f$ ), and $\omega N$ is the natural frequency of bubble oscillations. Since the damping constant and the natural frequency depend on the equilibrium bubble size, their contributions need to be integrated over the distribution, $f(R 0)$, for polydisperse mixtures. For the case of gas bubbles (without vapor), the damping constant and the natural frequency can be written as [64]

$$
2 \mu l p_{g 0} \omega^{2} R 0 / 2 c l
$$

$$
\begin{gathered}
\beta=+\Im\{Y\}+(17) \\
00 R^{2} 2 \rho l \omega R^{2} 1+(\omega R 0 / c l)^{2} \\
() \\
\omega^{2}=\Re\{Y\}-\quad+\omega^{2},(18)
\end{gathered}
$$


$\rho l R^{2} p_{g 0} R_{0} 1+(\omega R 0 / C l)^{2}$ 
where the first, second and third terms on the right-hand side of the damping constant stand for viscous, thermal and acoustic contributions, respectively. The quantity $\Upsilon$ is a function of the Peclet number $\left(\mathrm{Pe}=\omega R_{0}^{2} / \alpha_{T} ; \alpha \mathrm{T}\right.$ is the thermal diffusivity of the gas):

$$
\begin{aligned}
\Upsilon= & (\sqrt{ }) \cdot(19) \\
& \sqrt{ } 1-i 3\left(\bigvee_{g}-1\right) \mathrm{Pe}^{-1} \text { iPecoth iPe }-1
\end{aligned}
$$

The effective polytropic index in (13) for thermal behavior of the gas is therefore given by $\kappa=\Re\{Y\} / 3$. In the limit of $\mathrm{Pe} \rightarrow 0$, the thermal behavior is isothermal $(\kappa \rightarrow 1)$. Large values of $\mathrm{Pe}$, on the contrary, imply that the thermal boundary layer is thin compared to the bubble radius and therefore the bubble tends to behave adiabatically $\left(\kappa \rightarrow \gamma_{g}\right)$. For free oscillations of such large-sized bubbles, the frequency is well approximated by the Minnaert frequency [54]:

$$
\begin{gathered}
13 \gamma_{g p l 0} \\
\omega M=,(20) \\
R 0 \rho l
\end{gathered}
$$

where the effects of surface tension, heat transfer and acoustic radiation in (18) are ignored.

The phase velocity $c^{\text {ph }}$ and the attenuation $a^{\text {att }}$ are defined as [25]

$$
\begin{aligned}
& \mathrm{ph}^{\mathrm{ph}}=\mathfrak{R},(21) \\
& { }^{-1}{ }^{-1} \\
& a=-20\left(\log _{10} \mathrm{e}\right) \omega \mathfrak{I},(22)
\end{aligned}
$$

$\mathrm{Cm}$

where $a$ at $h a s$ the unit of decibels per length. The acoustic theory is known to overestimate attenuation under resonance [88], but the error associated with resonance can be deemphasized by including the broad bubble size distribution since the probability of finding bubbles of certain size under resonance is low among a wide spectrum of $R_{0}$ [32].

In Fig. 2, we examine the dispersion relation for linear waves in an air/water of void fraction $\alpha=0.001$ at standard temperature and pressure (STP; $20 \mathrm{C}, 101 \mathrm{kPa})$. The bubble size in the mixture is assumed lognormally distributed about $R^{\text {ref }}=$

with varying the standard deviation $\sigma$. Figure 2(a) shows the phase velocity in alone far above the resonant frequency (at which bubbles cannot respond to such high 
frequency excitation), the sonic speed of the mixture in a low frequency limit is given by [15]

$$
\begin{gathered}
\text { ph } \\
c=\lim _{c}=. \underset{f \rightarrow 0}{(23)} \operatorname{\rho \alpha m}(p l+B)+(1-\alpha) p l
\end{gathered}
$$

Thus, in the quasistatic regime (where all the phases are in dynamic equilibrium at all times), the sonic speed can be even smaller than the speed of sound in air, as in 

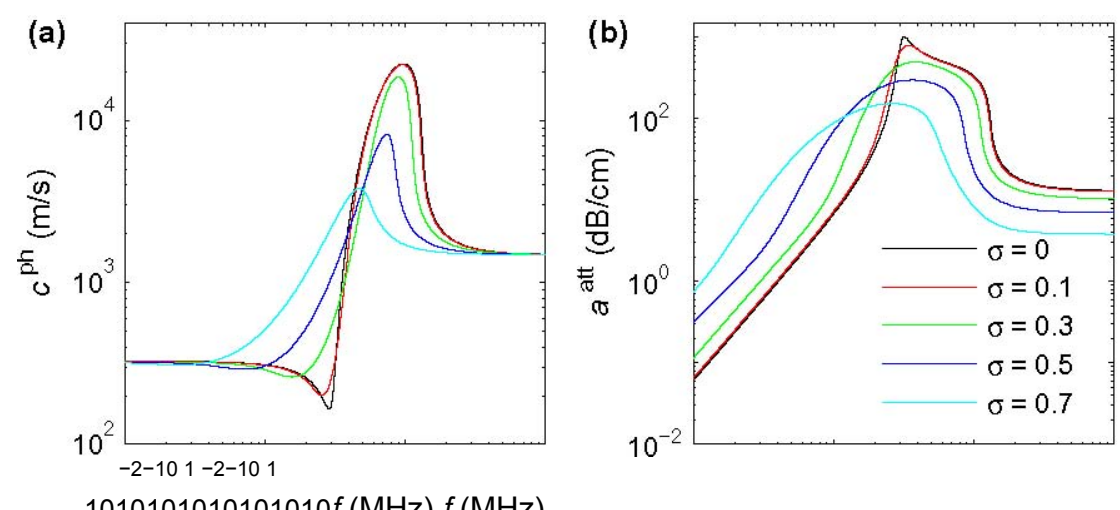

Fig. 2 Dispersion relation for linear waves in an air/water mixture of $\alpha=0.001$ at STP. The bubble size is assumed lognormally distributed about $R^{\text {ref }}=10 \mu \mathrm{m}$ with varying $\sigma$. The isothermal natural

Attenuation.

0 frequency for $R^{\text {ref }}$ is $0.29 \mathrm{MHz}$. (a) Phase velocity. (b)

this particular example. That is, the inclusion of a tiny amount of bubbles yields significant reductions in the fluid compressibility, while the fluid is yet dense. However, around the resonant condition, the bubble size distribution tends to have a striking impact on the phase velocity. If the mixture is monodisperse (i.e., $\sigma=0$ ) or the distribution is narrowly peaked (say, $\sigma=0.1$ ), the phase velocity is reduced as the external frequency increases to the resonant frequency, for the amplitude of bubble oscillations becomes larger and the mixture compressilibity is thus enhanced. If further increasing the frequency above the resonant frequency, the phase of the oscillations changes. Specifically, an increase in the ambient pressure leads to bubble expansion. Conceptually, the mixture becomes stiffer than the host liquid so that the phase velocity above the resonant frequency goes beyond the speed of sound in the liquid. On the other hand, for the case of broad size distributions, the abrupt transition across the resonant frequency is effectively eliminated due to the fact that having bubbles of certain size under resonance is less likely among a broud spectrum of the distribution.

Furthermore, it should be noted that the bubble size distribution increases the attenuation below the resonant frequency (see Fig. 2(b)). To interpret this trend, we consider linear bubble oscillations under sinusoidal excitation (without mutual interactions between the bubbles) [64],

$$
\begin{aligned}
& \varepsilon p l 0 \\
& \chi^{\prime \prime}+2 \beta \chi+\omega_{N}^{2} \chi=-\sin (\omega t),(24) \\
& \rho_{l} R^{2} \\
& 0
\end{aligned}
$$

where $\varepsilon$ is the infinitesimal amplitude of sinusoidally oscillating far-field pressure $(\mid \varepsilon$ $\mid \ll 1)$ and $\chi$ is the corresponding perturbed bubble radius: 
$R=R_{0}(1+\mathrm{X})$ 


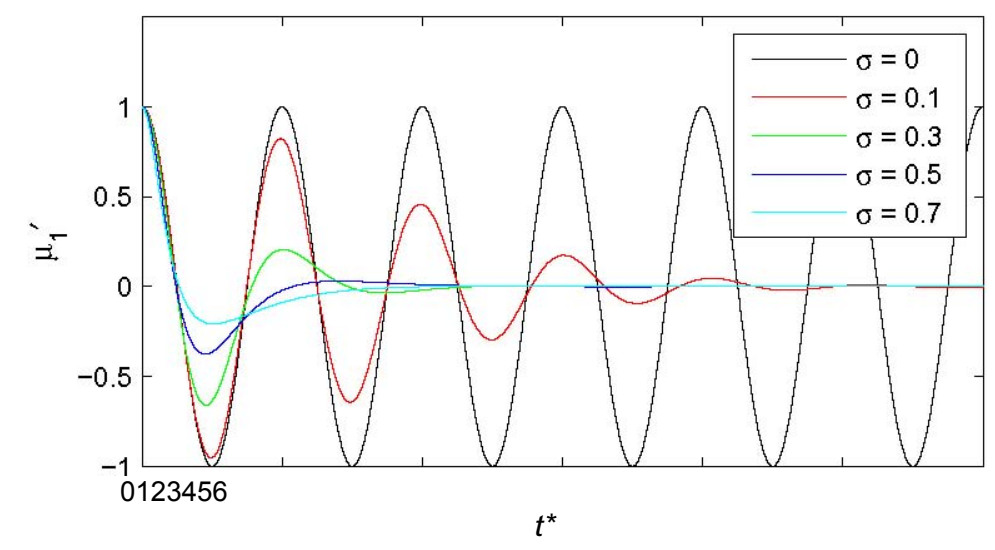

Fig. 3 Evolution of the first moment (35) as a function of time normalized by the Minnaert frequency $(\omega M / 2 \pi)$ for $R_{0}^{\text {ref }}$. The (inviscid) bubbles are set into linear oscillations according to a stepwise change in the farfield pressure. The bubble size is assumed lognormally distributed with the standard deviation $\sigma$.

The particular solution to the linearized problem (24) is

$$
-\varepsilon p l 0
$$

$$
\begin{gathered}
\chi_{p}=\geqslant \sin (\omega t+\varphi),(27){ }_{2} \\
\rho l R^{2} \omega_{N}^{2}-\omega^{2}+4 \beta^{2} \omega^{2}
\end{gathered}
$$

0

where the phase shift $\varphi$ is given by

$$
\begin{aligned}
& \varphi=\cos \\
& \omega_{2} 2-\omega_{2} \\
& \omega^{2}-\omega^{2}+4 \beta^{2} \omega^{2}
\end{aligned}
$$

Noting that the damping constant and the natural frequency depend on the bubble size in the quasistatic regime, the phase shift can also vary with different values of $R 0$ so that there may arise cancellations due to the different phases among the different-sized bubbles. Hence, (collective) volume oscillation of a polydisperse cloud is deemphasized due to the phase cancellations. In other words, the phase cancellation effect can be regarded as an apparent damping of the mixture-averaged dynamics and is augmented as the distribution broadens [2]. This additional damping associated with polydispersity may account for the observation at low frequency in Fig. 2(b).

The phase cancellation effect appears also in free oscillations in one-way-coupled flow, which may be modeled by replacing the sinusoidal excitation term on the right-hand side of (24) with stepwise forcing; namely 
$\ddot{\chi}+2 \beta \chi+\omega_{N}^{2} \chi=-H(t),(29){ }^{\rho} l^{R^{2}} 0$ 
where $H(t)$ is the Heaviside step function. If the bubbles are initially stationary (i.e., $\chi$ $=0$ and $\chi=0$ at $t=0$ ), the solution to (29) is then given by

[]

$\varepsilon p_{10} \Omega-\mathrm{e}^{-\beta t} \Omega \cos (\Omega t)-\beta \sin (\Omega t)$

$\mathrm{X}=-,(30)$

$\rho l R_{0}^{2} \Omega \beta^{2}+\Omega^{2}$

where $\Omega=\left(\omega_{v}^{2}-\beta{ }^{2}\right)^{1 / 2}$. For the inviscid case $(\beta=0)$, the bubbles keep oscillating without any damping and the solution (30) is simplified to

[]

$\varepsilon \operatorname{plocos}(\omega N t)-1 \quad \chi=\varepsilon 3 \gamma_{g} \cos t R 0 \quad 3 \gamma_{g} p l 0 \rho l-1$,

$\chi=.(31)$

$\rho_{l} R_{0}^{2} \omega^{2}$

$N$

Moreover, if the natural frequency is approximated by the Minnaert frequency $\omega \mathrm{M}$ in (20), the inviscid solution further reduces to

which can be redefined as a normalized perturbation from the new equilibrium:

()

$3 \gamma_{g} \&$ t $3 \gamma_{g} p l 0$

$\chi=x+=\cos .(33)$

$\varepsilon 3 \gamma_{g} R 0 \rho l$

To observe the dynamics of clouds in which the bubbles are freely oscillating according to (33), we define the moments of the (perturbed) bubble radius with respect to a smooth bubble size distribution $f(R 0)$ :

।

$\infty[]_{j}$

$\mu_{j}(t)=\mathrm{X}(t ; R 0) f(R 0) \mathrm{d} R 0 .(34)$

0

For example, $\mu$ stands for the mean bubble radius. It follows from the RiemannLebesgue lemma [9] that the first moment vanishes as $t \rightarrow \infty$, provided that the 
integral $\left|f\left(R_{0}\right)\right| \mathrm{d} R 0$ is bounded:

${ }^{\infty}{ }^{3} \gamma_{g} p_{l 0}$

,

$\mu_{1}(\infty)=\lim \cos f\left(R_{0}\right) \mathrm{d} R 0=0$. (35)

$t \rightarrow \infty R_{0} \rho_{l}$

That is, bubble oscillations eventually reach a statistical equilibrium at which the different-sized bubbles oscillate totally out of phase and the polydisperse bubble cloud can thus be considered to be stationary, regardless of inviscid bubble oscillations. The existence of the statistical equilibrium is numerically verified in Fig. 3

where the moment $\mu_{1}$ is evaluated using the lognormal function (8). While (inviscid) oscillations continue in the monodisperse mixture $(\sigma=0)$, the inclusion of bubble size distributions eventually yields a time-invariant value. Furthermore, the broader the distribution, the more quickly cancellation between bubbles at different phases of their oscillation cycles is achieved. If the distribution is sufficiently broad, the moment can converge to a stationary state, due to apparent damping associated with the strong cancellation effect, only within a couple of oscillation periods for the probable size. 
Nonlinear oscillations of inviscid bubbles in one-way-coupled flow can also be shown to reach a statistical equilibrium and the apparent damping associated with polydispersity may dominate over physical dampings, in the averaged sense, if the distribution is sufficiently broad (for details see $[3,24,75]$ ). In the next section, we simulate one-dimensional shock propagation based on the continuum model and show that the phase cancellation effect comes into play in two-way-coupled poly-disperse flows as well.

\section{Simulation of Averaged Shock Dynamics}

\subsection{Numerical Method}

Because shocks in bubbly flows often contain oscillatory structures due to bubble dynamics, we favor the properties of high-order accurate resolution in complex smooth structures as well as shock capturing and robustness. Here, we select a finite-volume (FV) weighted essentially non-oscillatory (weighted ENO or WENO) scheme [52, 72], which has proven to be stable and accurate for shock computations in various examples, together with monotonicity preserving bounds [7]. ENO reconstruction [38] is based on adaptive stencils in the sense that interporation across discontinuities is automatically avoided. WENO schemes consist of a convex conbination of all the ENO candidate stencils for more efficient and accurate evaluations. To guarantee entropy solutions, it is safe to implement the WENO procedure in characteristic space [67].

The system of the governing equations in one dimension can be written as

$\partial \mathbf{q} \partial \mathbf{f}(\mathbf{q}) \partial \mathbf{f}^{s}(\mathbf{q})$

$+=+\mathbf{s}(\mathbf{q})$. (36)

$\partial t \partial x \partial x$

The column vectors (conserved variables, numerical fluxes, and bubble-dynamic 
where $u$ is the $x$-component velocity, $\phi$ represents the bubble-dynamic variables such as $R$ and $R^{\circ}$, and the superscript $s$ denotes sources that vanish in the equilibrium state. Given a computational cell $\left[x_{i}-1 / 2, x_{i}+1 / 2\right]$ where $i$ denotes the grid index, the system (36) is discretized in FV fashion:

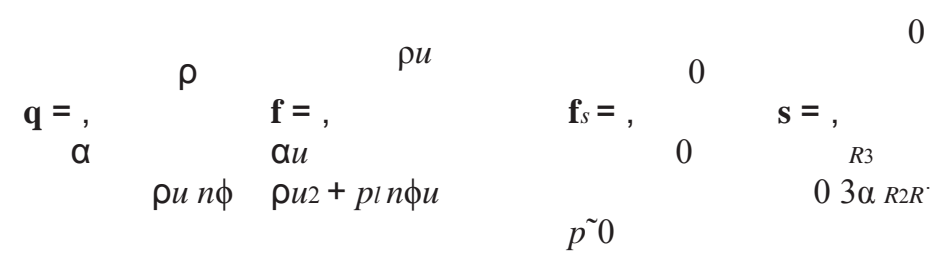




$$
\begin{aligned}
& \frac{\mathrm{dq}^{-} i \mathbf{f}_{i+1 / 2}-\mathbf{f}_{i}-1 / 2 \underline{i-1 / 2}}{\mathbf{f}_{i+1 / 2}-\mathbf{f}_{s}} \\
& =-++\mathbf{s}^{-}{ }_{i},(38) \\
& \mathrm{d} t \Delta x_{i} \Delta x_{i}
\end{aligned}
$$

where $\Delta x_{i}$ is the cell width $\left(x_{i+1 / 2}-x_{i-1 / 2}\right)$ and the overbar denotes the cell-averaged quantities. In the FV method, $\mathbf{q}_{i}^{-}$is reconstructed at each side of the cell edge and the numerical flux $\mathbf{f}_{i+1 / 2}$ is determined from a local Riemann problem. Here, we implement the fifth-order monotonicity-preserving FV-WENO scheme in the characteristic space for reconstructing the cell-edge conserved variables and then calculate the numerical flux using the HLLC approximate Riemann solver $[84,83]$ that automatically satisfies the entropy condition. The HLLC Riemann solver restores contact waves that the HLL Riemann solver [39] ignores, and thus gives better resolutions of the contact discontinuities.

Once the HLLC fluxes and the sources are determined, the system (38) in semi-discrete form can be integrated in time. In the examples in Sect. 3.3, a third-order TVD Runge-Kutta scheme $[37,73]$ is used to integrate both the convective terms and the bubble-dynamic sources. Such an unsplit integration method works for weak shocks, for the bubble-dynamic sources are not very stiff. It is instructive to note, however, that if the system is stiffer in particular for cases with strong shocks or cavitation bubbles, one may need to apply time-step splitting techniques $[35,51,83]$ or implicit methods $[23,80]$ for stable time integration.

In Sect. 3.3, the FV-WENO scheme together with the HLLC Riemann solver is used to compute the numerical fluxes and the system is inetgrated in time using the unsplit method. The computational grid is uniform with $\Delta x=R^{\text {ret }}$. For the polydis

$$
0
$$

perse case, the moments (7) associated with the distribution of equilibrium bubble sizes are evaluated using Simpson's rule with 101 quadrature points. This method has been shown to be accurate enough to resolve wave dispersion in a wide range of frequency. Moreover, a comparison to the experiment [45] validates the continuum approach to predict mixture-averaged dynamics for weak shocks. For further infomation, see [3].

\subsection{Steady Shock Relations}

The simulation of shock propagation requires the steady shock relations to be used as initial conditions. While shocks in single phase flow have infinitesimal thickness (in a continuum sense), the thickness of shocks in bubbly flow becomes finite due to bubble-dynamic relaxation. In front of the shock, the bubbles are in equilibrium at $(R 0$, $\left.T_{0}, p l 0\right)$ where $T_{0}$ is the (undisturbed) liquid temperature. Far downstream of the shock front, the bubble dynamics are finally damped out and the bubbles are once again in equilibrium at $(R H, T 0, p l H)$ where $R H$ is the new equilibrium radius corresponding to the shock pressure $p l H$. The specification of $T_{0}$ in the final equilibrium state implies that the bubble temperature eventually returns to the liquid temperature due to heat 
conduction across the bubble wall. Now, the one-dimensional conservation laws for mass, momentum and bubble number are written in a coordinate system moving with the constant shock velocity $U_{s}$ : 
$\mathrm{d} \rho u=0,(39)$

$$
\mathrm{d} x^{\prime} \quad \mathrm{d}{ }{ }_{2}{ }_{+p_{l}-}
$$

$(\rho u p)=0,(40)$

$$
\mathrm{d} x,
$$

$\mathrm{d} n u$

$$
=0,(41) \mathrm{d} x
$$

where $u$ is the velocity in the coordinate system with $x=x^{-} U$ st. Integrating (39) to (41) from upstream to far downstream, it transpires that, independent of the detailed shock structure,

$$
\begin{gathered}
\left.-\rho н u_{H}=\rho_{0} U_{s},(42)\right)^{\prime} 2 \\
\rho н u_{H}+p l H=\rho 0 U_{s}^{2}+p l 0,(43) \\
- \text { пни }=(44) \\
H^{n} 0{ }^{U} s
\end{gathered}
$$

where the subscripts 0 and $H$ denote upstream and downstream quantities, respectively, and $\rho_{0}=\left(1-\alpha_{0}\right) \rho_{l 0}$ and $\rho_{l H}=\left(1-\alpha_{H}\right) \rho_{l H}$.

The new equilibrium bubble radius $R_{H}$ is related to the shock pressure $p_{l H}$ :

$$
()()-3
$$

$$
\begin{array}{r}
2 \Upsilon R_{H} 2 \Upsilon \\
p_{l H}=p_{l 0}-p_{v}++p_{v}-\quad,(45)
\end{array}
$$

$R_{0} R_{0} R_{H}$

where the bubbles are assumed to behave isothermally so that the bubble temperature finally returns to $T 0$. From (42) and (44), we write the bubble number density far behind the shock as

$$
\begin{gathered}
()_{1 / m}-1 \\
\frac{p l 0}{n H}=\left(1-\alpha_{0}\right)+{ }_{0 R^{3}} .(46) \\
p l H_{l H}+B 3
\end{gathered}{ }^{H}
$$

From (42) and (43), the steady shock speed becomes 
plH $-p l 0$

$U_{s}=(),(47)$

$\rho_{01-}$

and the induced velocity far downstream of the shock front is then given by ()

$\rho 0$

$u H=u_{H}+U_{s}=1-U_{s .}(48)$

$\rho H$

It can be shown that the shock speed (47) approaches the sonic speed (23) if the shock strength is infinitesimal. 


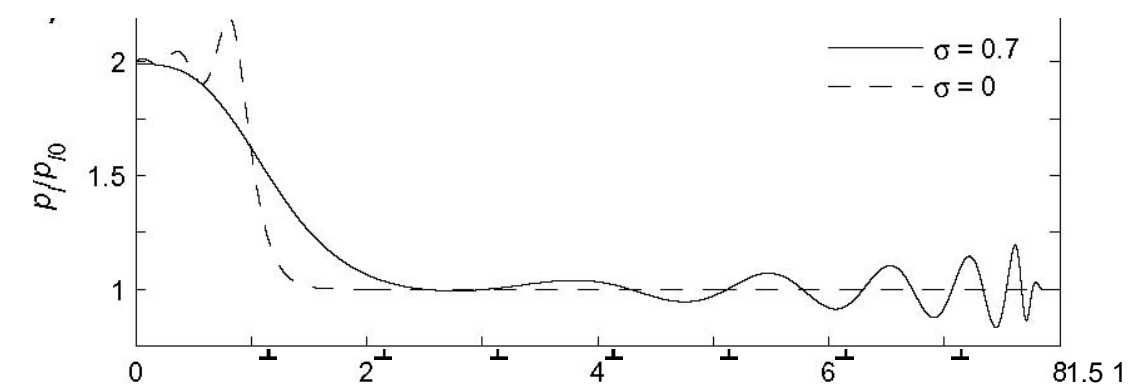

(a)

$/ p$
$\times 10^{-2}$

$a$

0.3

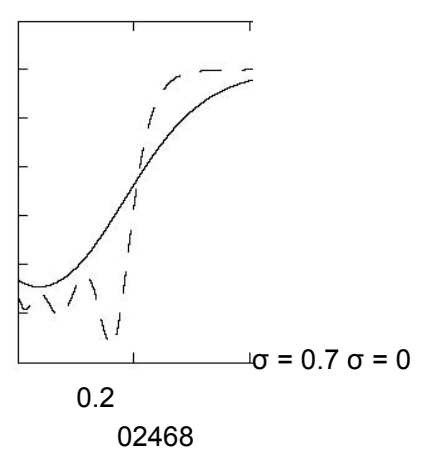

(c) 1.1

(b)

1 
$x(\mathrm{~mm})$

Fig. 4 Spatial evolution of the unsteady shock propagation, $5.2 \mu$ s after the steady shock relations are imposed at $x=0$, through an air/water mixture of $\alpha_{0}=0.005$. The equilibrium bubble radius is lognormally distributed about $R^{\text {ref }}=10 \mu \mathrm{m}$ with the standard deviation $\sigma .(\mathbf{a})$ Averaged liquid

pressure. (b) Void fraction. (c) Bubble radius with different equilibrium sizes.

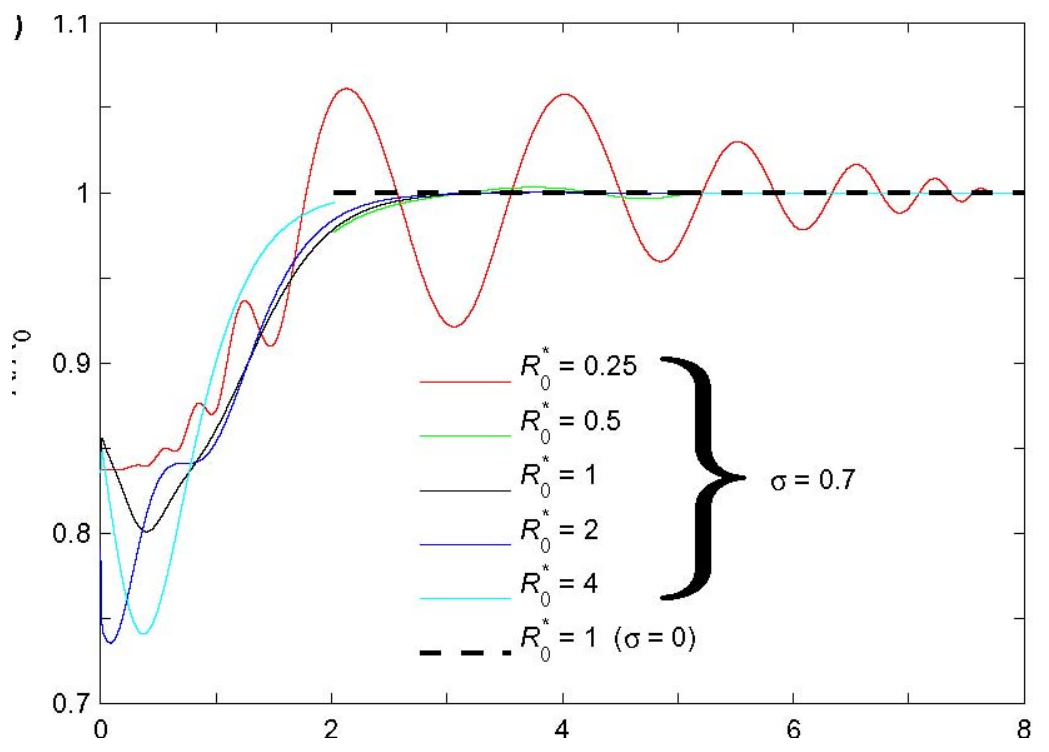



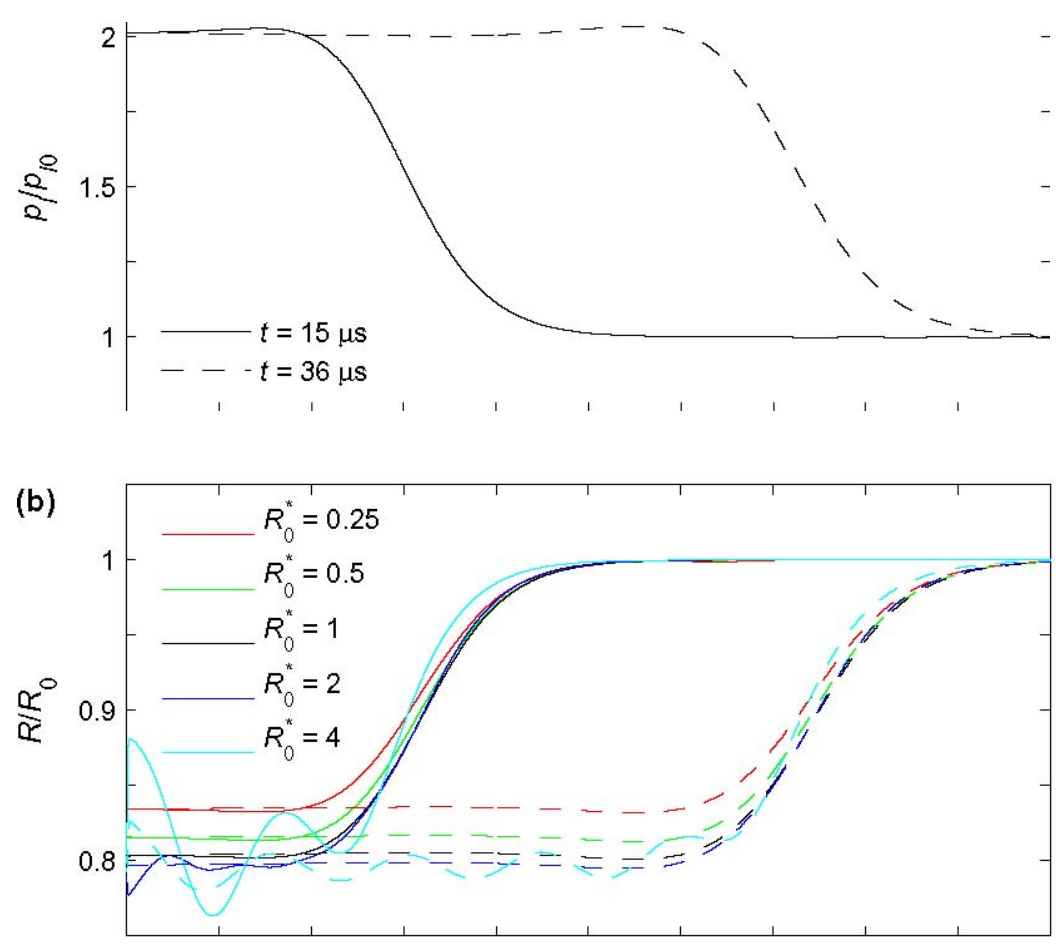

(a)

$$
\begin{gathered}
t=15 \mu \mathrm{s} t=36 \mu \mathrm{s} \\
0246810
\end{gathered}
$$

Fig. 5 Spatial evolution of the shock propagation, $15 \mu$ s (solid lines) and $36 \mu$ s (dashed lines) after the steady shock relations are imposed at $x=0$, through the polydisperse mixture of $\alpha 0=0.005,{ }_{R}$ ref

$=10 \mu \mathrm{m}$, and $\sigma=0.7$. (a) Ensemble-averaged liquid pressure. (b) Bubble radius with different equilibrium sizes.

\subsection{One-Dimensional Shock Propagation}

Here, we consider unsteady and steady shock propagation through a polydisperse bubbly liquid. As a representative example, we simulate shock propagation in an air/water mixture of void fractions below $\alpha_{0}=0.005$ at STP. The equilibrium bubble size is assumed lognormally distributed with varying the standard deviation $\sigma$. The shock pressure is set to $p l H=2 p l 0$. The steady shock relations in Sect. 3.2 are initially imposed by a diaphragm at $x=0$. Diffusive effects on the dynamics of single bubbles (i.e., heat and mass diffusion at the bubble wall; see Sect. 2.2) are evaluated using the reduced-order model of Preston, Colonius, and Brennen [63], which is accurate for the case of micron-sized air bubbles in water. We judge steadiness by observing the first 
peak of pressure oscillations due to bubble dynamics; 
$x(\mathrm{~mm})$

Fig. 6 Spatial evolution of the averaged liquid pressure for steady shock propagation through an air/water mixture of $\alpha_{0}=0.005$. The equilibrium bubble radius is lognormally distributed about ${ }_{R}$ ref

$=10 \mu \mathrm{m}$ with $\sigma$ ranging from 0 to 0.7 . The position where the pressure is $(p l 0+p l H) / 2$ is set 0 at $x=0$.

if the peak pressure is unchanged, the shock propagation is considered to be in a steady state. Note that we limit our attention to the case of weak shocks in a dilute liquid in order to avoid issues associated with bubble fission [16, 29] and direct bubble interactions $[35,70]$.

In Fig. 4, we examine unsteady shock propagation in an air/water mixture of $\alpha_{0}=$ 0.005 and $R^{\text {ref }}=10 \mu \mathrm{m}$ at STP; the mixture is monodisperse $(\sigma=0)$ or polydisperse

$0(\sigma=0.7)$. In addition to the averaged liquid pressure and void fraction fields, the spatial evolution of the bubble radius for different equilibrium sizes $\left(R_{0}^{*}=0.25,0.5,1,2,4\right)$ is plotted to visualize the individual bubble dynamics. It follows from the pressure field (evidently for the polydisperse case) that high-frequency waves precede the primary shock wave and propagate essentially with the speed of sound in (pure) water. However, these precursory waves do not perturb the void fraction field (Fig. 4(b)), for most bubbles (excluding very small bubbles) do not respond to such high-frequency forcing (Fig. 4(c)). While the precursory pressure waves in the monodisperse mixture is damped out, those in the polydisperse mixture are still on the decay. This is because the bubble size distribution decreases the attenuation of high-frequency acoustic waves as demonstrated in Fig. 2(b). It is also interesting to note that oscillatory shock structure is obtained in the monodisperse mixture but not in the polydisperse mixture in which the different-sized bubbles oscillate with different phases in the neighborhood of the shock front. To see the details of the polydisperse case, Fig. 5 presents the spatial evolution of the shock propagation at two different times at which the larger-sized bubbles still show oscillations with less effective damping. Despite the (undamped) bubble oscillations, the shock profile in the averaged pressure field seems unchanged during this period; the shock propagation can thus be said to reach a steady state. Because the different-sized bubbles oscillate with different phases as in the case of linear waves 



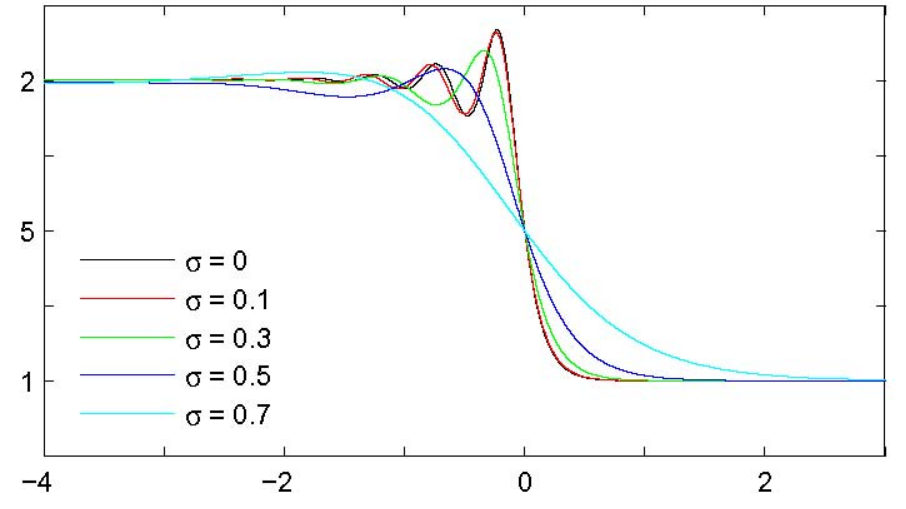




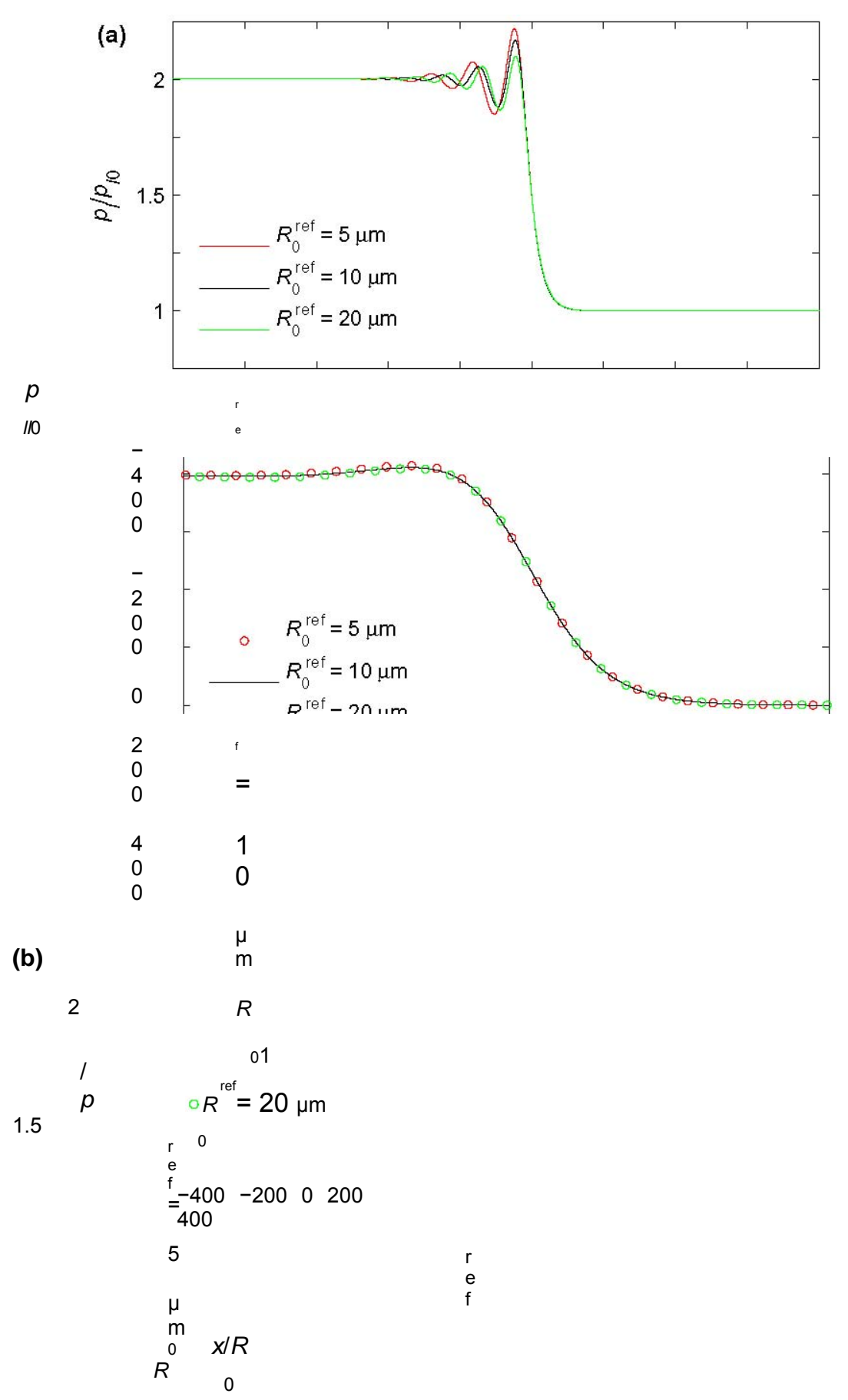

Fig. 7 Effect of the probable bubble size $R^{\text {ref }}$ on steady shock structure in an air/water mixture of

$\alpha_{0}=0.005$. The position where the pressure is $(p l 0+p l H) / 2$ is set at $x=0$. (a) Monodisperse case $(\sigma=$ $0)$. (b) Polydisperse case $(\sigma=0.7)$. 
(Sect. 2.3), the phase cancellations associated with the distribution yield an apparent damping of the mixture-averaged shock dynamics, which can be effective enougth to make the averaged dynamics insensitive to the behavior of individual bubbles as in this example with a broad distribution. To be specific, the phase cancellations in a polydisperse bubble cloud occur locally and bubble oscillations reach a statistical equilibrium (defined in free oscillations of inviscid bubbles in one-way-coupled flow; see Sect. 2.3) shortly after the shock passage, so that the polydisperse cloud does not oscillate in the averaged sense. Hence, this collective effect leads to such a smoothed shock profile.

The steady shock structures in the averaged pressure field are plotted in Fig. 6; the simulation conditions are the same as in Figs. 4 and 5 with $\sigma$ ranging from 0 to 0.7 , but steady solutions are now presented. If the distribution is narrowly peaked, oscillatory shock structure is obtained still in the steady state. For the distribution to 


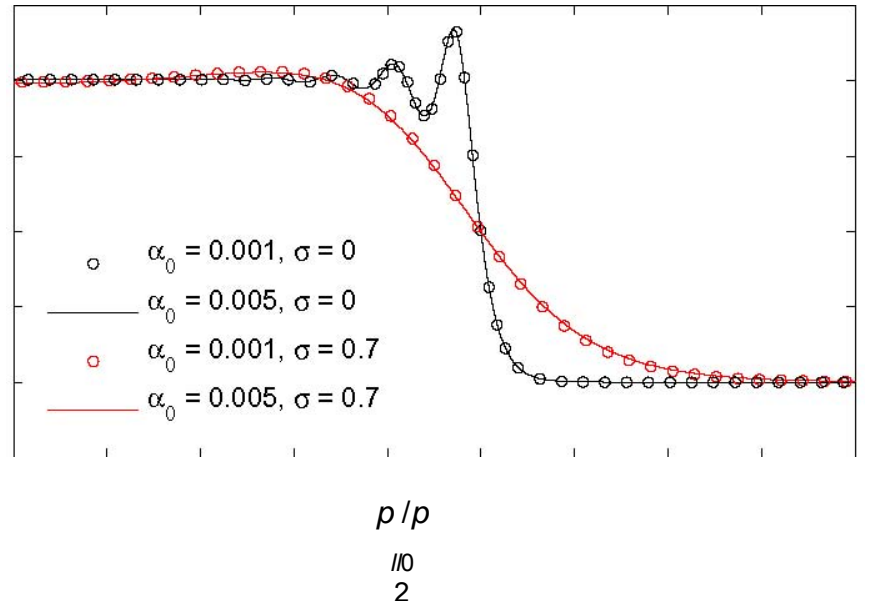

1.5

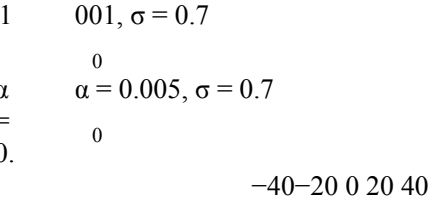

$$
x \omega_{N}\left(R_{0}{ }^{\mathrm{ref}}\right.
$$

Fig. 8 Effect of the initial void fraction $\alpha_{0}$ on steady shock structure in an air/water mixture of ${ }_{R}$ ref

$=10 \mu \mathrm{m}$. The position where the pressure is $\left(p_{l 0}+p_{l H}\right) / 2$ is set at $x=0$.

further broaden, on the contrary, the averaged shock structure becomes less oscillatory and the pressure rise tends to be more gradual. Namely, the broader distribution results in the more effective apparent damping because the phase cancellation effect is augmented with increasing $\sigma$. If the distribution is sufficiently broad (say, $\sigma=0.7$ ), the shock profile is smoothed out, indicating the quasistatic behavior of a polydisperse bubble cloud in spite of individual bubble dynamics. Such a smoothed shock profile in a polydisperse mixture is reported in the experiment of Beylich and $\mathrm{G}^{*}$ ulhan [11].

To further assess the effect of polydispersity, we continue a parametric study on the shock propagation. In Fig. 7, different values of the probable bubble size ${ }_{(R}$ ref

$=15 \mu \mathrm{m}, 10 \mu \mathrm{m}, 20 \mu \mathrm{m})$ are selected to examine its impact on the averaged

0 shock dynamics in an air/water mixture of $\alpha_{0}=0.005$ for both monodisperse and polydisperse cases. The intent is to compare the apparent damping associated with 
polydispersity to physical damping (mainly due to thermal dissipation) that depends on the value of $R^{\text {ref }}$. The spatial coordinate is normalized by $R^{\text {ref }}$ for the compari

00 son. For the monodisperse case (with no phase cancellation effect), the first peak in the pressure oscillations decreases with increasing $R^{\text {ref }}$; the single-bubble-dynamic

0 damping has an impact

on the averaged shock structure. However, the inclusion of the broad bubble size distribution with $\sigma=0.7$ leads to the observation that the shock profiles with different values of $R_{0}^{\text {ref }}{ }_{0}$ coincide in the normalized coordinate. This means that the dynamics of the polydisperse bubble cloud are insensitive to the physical dissipation in bubble oscillations, which is overwhelmed by the apparent damping associated with the broad distribution. Finally, the effect of the initial void fraction, $\alpha$, on the shock simulation with ${ }_{R}$ ref

$=10 \mu \mathrm{m}$ is explored in Fig. 8. Two different values $\left(\alpha_{0}=0.001,0.005\right)$ are 0 chosen; the corresponding sonic speeds of the mixture (23) in the low frequency limit are $312 \mathrm{~m} / \mathrm{s}$ and $142 \mathrm{~m} / \mathrm{s}$, respectively. In the coordinate normalized by the characteristic length $c / \omega_{N}$ where $c$ is the sonic speed (23) and $\omega_{N}$ is the isothermal natural frequency for $R^{\text {ref }}$, the averaged shock structures coincide for both monodis 
0 perse and polydisperse cases. That is, a choice of the initial void fraction simply changes the propagation speed but the solutions remain similar.

\section{Shocks in a Mixture-Filled Deformable Tube}

\subsection{Quasi-One-Dimensional Conservation Laws}

In the preceeding section, particular attention has been paid to the effect of polydispersity on the mixture-averaged dynamics for weak shocks. Now, we focus on nonlinear effects attributed to compressible bubbles in particular for strong shocks. One of challenges for creating strong shocks in a fluid-filled tube is related to tube deformation that can be regarded as additional compressibility in the shock propagation [89]. The simplest way to tackle this issue is to apply quasi-one-dimensional analysis so as to account for the tube deformation. In what follows, we include the effect of fluid-structure interaction (FSI) in the ensemble-averaged bubbly flow equations presented in Sect. 2.1.

Let $A$ be the internal cross-sectional area of a (fluid-filled) cylindrical tube. Since the effect of tube dynamics on linear wave speeds is negligible in a low-frequency limit [74, 82], small tube deformation may be modeled by [71]

$2 a_{0}$

$$
A=A 01+\left(p_{l}-p l 0\right),(49)
$$

Eh

where $a$ is the mid-plane tube radius, $h$ is the wall thickness, $E$ is Young's modulus of the tube material, and the subscript 0 denotes the initial (undisturbed) values. This quasistatic relation can be obtained from the balance between liquid pressure $p_{l}$ and stress associated with the hoop strain $\varepsilon \theta$ :

$$
\varepsilon \theta=\stackrel{a_{0}}{(p l-p l 0)} \cdot(50)
$$

From a conventional control volume anlysis, the quasi-one-dimensional versions of the mass, momentum, and bubble number conservation laws are written as

$$
\partial \rho A \partial \rho u A
$$$$
+=0,(51)
$$

$\partial t \partial x \partial \rho u A \partial[] \partial A$

$$
+\rho u^{2} A+\left(p l-p^{\sim}\right) A=p l,(52)
$$

$\partial t \partial x \partial x \partial n A \partial n u A$

$$
+=0,(53)
$$


where quantities are cross-sectionally averaged. With the quasistatic relation (49) for tube deformation, the momentum equation (52) is rewritten as

$$
\begin{aligned}
& \partial \rho u A \partial \mathrm{Aloa}_{2} \\
& +\rho u^{2} A+\left(p l-p^{\sim}\right) A-\quad p=0 . \text { (54) } \\
& \quad \partial t \partial \times E h^{1}
\end{aligned}
$$

For future use, we introduce the sonic speed of the mixture, in a low frequency limit, that accounts for the structual compressibility as well as the fluid compressibility [14]:

$$
\begin{aligned}
& )_{-1 / 2} \\
& 1 \mathrm{~d} \rho A c \\
& C J==\text {. (55) } \\
& A \mathrm{~d} p l 1+\xi
\end{aligned}
$$

Here, $c$ is the sonic speed (23) of the mixture (without FSI) and $\xi$ determines the extent of fluid-structure coupling defined as

$$
\xi=, \begin{gathered}
2 K a_{0} \\
E h
\end{gathered}
$$

where $K$ is the bulk modulus of the mixture that depends on the void fraction $\alpha$ :

$$
\begin{gathered}
11-\alpha \alpha \\
=+.(57) \\
\text { K Kl Kg }
\end{gathered}
$$

The bulk modulus of the gas phase (bubbles) is written as $K_{g}=\mathrm{K} p l$ where $\kappa$ is the polytropic index that approaches unity in the quasistatic limit; the bulk modulus of the (Tait) liquid is given by $K_{l}=m\left(p_{l}+B\right)$. The wave speed (55) is identical to that of Kobori et al. [47]. For rigid tubes $(E \rightarrow \infty)$, this expression coincides with the mixture sonic speed (23). In the dilute limit $(\alpha \rightarrow 0)$, this approaches the so-called Korteweg-Joukowsky wave speed [43, 48]:

$$
\begin{aligned}
& C l \\
& C J
\end{aligned} \rightarrow \text { ClJ }=.(58) 1+\xi l
$$

Here, $\xi_{l}$ is the FSI parameter for the liquid:

$$
\xi_{l}=\text {. (59) }
$$

\subsection{FSI Shock Theory}

Eh

Following the same procedure in Sect. 3.2, one can derive the steady shock relations 
corresponding to the quasi-one-dimensional conservation laws. Integrating Eqs. (51) to (53), in a frame of moving with the shock speed $U_{s}$, from upstream (denoted by the subscript 0 ) to far downstream (denoted by the subscript $H$ ), we find 


$$
\begin{gathered}
\left.-\rho н u_{H} A H=\rho_{0} U_{s} A 0,(60)\right)^{\prime} 2 \\
\rho н u_{H} A H+g(p l H)=\rho_{0} U_{s}^{2} A 0+g(p l 0),(61) \\
-n н u_{H} A H=n_{0} U_{s} A 0,(62)
\end{gathered}
$$

where $u$ is the velocity measured in a coordinata system moving with $U_{s}$ and

$$
\begin{aligned}
& { }^{2 p}{ }_{0}^{a} 0 \quad{ }^{A} 0{ }^{a} 02 \\
& g\left(p_{l}\right)=A_{01}-p_{l}+p_{l} \cdot(63) \\
& \text { Eh Eh }
\end{aligned}
$$

The new equilibrium bubble radius $R_{H}$ is also determined from the isothermal relation (45). If one ignores vapor pressure and surface tension, it is simply given by

$$
()-3
$$

$$
p l H=p l 0 .(64)
$$

With this simple relation, the bubble number density (46) at $p l H$ is

()$_{l / m}-1 p l 0+B p l 0 n H=n_{0}\left(1-\alpha_{0}\right)+\alpha_{0},(65) p l H+B p l H$

and the void fraction at $p l H$ is

$$
\begin{aligned}
& ()_{1 / m}-11-\alpha_{0} \underline{p l H p l 0}+\mathrm{B} \\
& \alpha H=1+.(66) \alpha_{0} p l 0 p l H+B
\end{aligned}
$$

From (60) and (61), the steady shock speed becomes

$$
\begin{gathered}
\frac{g(p l H)-g(p l 0)}{U_{s}=(),(67)} \\
\rho 0 A 01-{ }^{\rho 0 A 0}
\end{gathered}
$$

and the induced velocity far downstream of the shock front is then given by ()

$$
\rho_{0} A_{0}
$$$$
u H=1-U_{s},(68)
$$$$
\text { }
$$

where $\rho_{H}=\left(1-\alpha_{H}\right) \rho_{l H}$. If the shock strength is infinitesimal $\left(p_{l H} \rightarrow p l 0\right)$, the shock 
speed (67) approaches the Korteweg-Joukowsky wave speed (55). Consequently, the shock Mach number may be defined as

$$
\frac{U_{s}}{M_{s}}=\text {. (69) } C J
$$

These relations reduce to the steady shock relations in Sect. 3.2, provided that the tube material is rigid $(E \rightarrow \infty)$. 


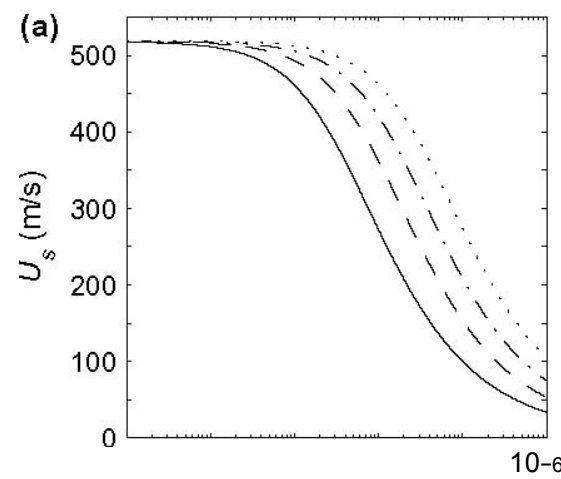

(b)

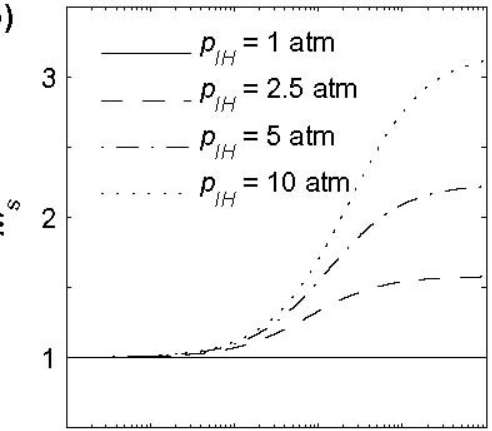

$10-6$ 10-4 10-2

a

$10-610-410-2$

Fig. 9 Effect of gas-phase compressibility on steady shock propagation through a fluid-filled de-formable tube, with varying the initial void fraction $\alpha$. The FSI parameter is set to $\xi_{l}=7.29$. (a) Shock speed. (b) Shock Mach number.

Figure 9 presents the steady shock relations for the case of bubbly water with the FSI parameter $\xi_{l}=7.29$, where the value of $\xi_{l}$ is determined from the properties of the tube and water used in the experiment [4] to be described in Sect. 4.3. For simplicity, we ignore the effects of vapor pressure and surface tension. In this figure, the shock speed and the corresponding shock Mach number are plotted as a function of the initial void fraction, with varying the shock pressure $p_{l H}$. The case of $p_{l H}=1 \mathrm{~atm}$ means the linear wave case, in which the shock speed (67) coincides with the sonic speed (55). At $\alpha_{0}=0$, the linear wave speed is given by the Korteweg-Joukowsky velocity $(C l J=$ $518 \mathrm{~m} / \mathrm{s}$ ), which is reduced, due to the structural compressibility, from the sonic speed of pure water. It follows from Fig. 9(a) that even a tiny amount of bubbles lead to a substantial reduction in the shock speeds. Moreover, unless the void fraction is extremely small, the finite shock strength yields a significant deviation from the linear wave speed due to the nonlinearity associated with the compressibility of bubbles. As a result, the shock Mach number increases with increasing $\alpha 0$ as seen in Fig. 9(b). Finally, we note that the shock Mach numbers are close to unity for the case of water alone $\left(\alpha_{0}=0\right)$ because pressure perturbations up to several hundred atmospheres remain very weak $[40,55]$. In what follows, we report briefly on the experiment [4] to validate the observation regarding the gas-phase nonlinearity from the FSI shock theory.

\subsection{Water-Hammer Experiments}

The experiments [4] were performed to measure coupled stress waves propagating in a deformable tube filled with fluids. The experimental setup (Fig. 10(a)) consists

of a polycarbonate tube $\left(E=2.13 \mathrm{GPa}, \rho_{s}=1200 \mathrm{~kg} / \mathrm{m}, a_{0}=3.5 \mathrm{~h}=22.2 \mathrm{~mm}\right)$ 
Steel_projectile

Air_outlet_

Polycarbonate_tube

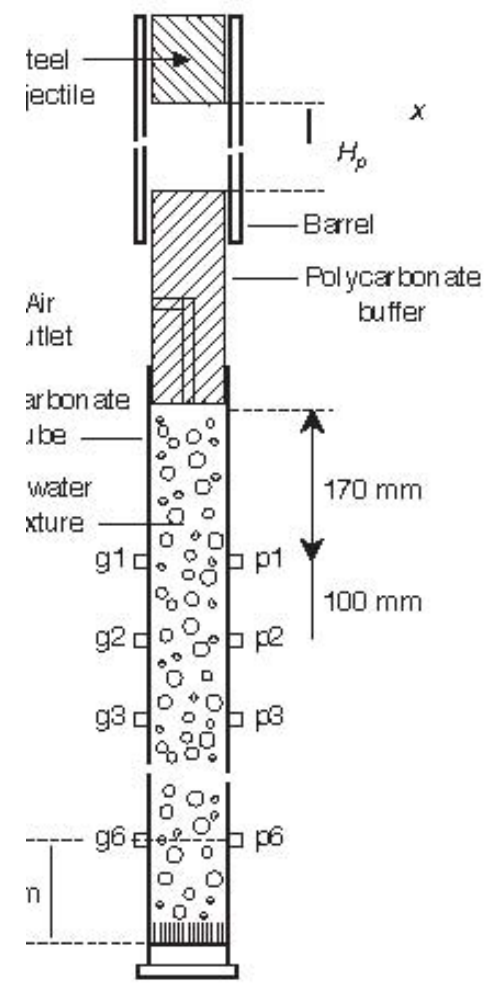

Air/water_mixture

(c)

g1_

g2

g3

g6 
Fig. 10 Water-hammer experiment for generating a shock through a fluid-filled tube. (a) Schematic of the experimental setup. The shock evolution is detected using strain gauges (g1 to g6) and pressure transducers (p1 to p6). (b) An example of the image of injected air bubbles with $\alpha 0=0.0081$. (c) The collapsing bubbles due to shock loading with $H_{p}=2 \mathrm{~m}$.

containing an air/water mixture inside. A $1.5 \mathrm{~kg}$ cylindrical steel projectile starts to fall $2 \mathrm{~m}$ above the top of the tube and then impacts a $0.46 \mathrm{~kg}$ polycarbonate buffer inserted into the tube. Stress waves in the tube material are measured using six strain gauges $\mathrm{g} 1$ to g6 (placed at intervals of $100 \mathrm{~mm}$ along the tube and oriented in the hoop direction); fluid pressures on the inner tube wall are measured using six pressure transducers $\mathrm{p} 1$ to $\mathrm{p} 6$, which are located at the opposite side of g1 to g6.

The bubbles are created using a bubble generator that consists of an aluminium plate and capillary tubes of inner diamater $20 \mu \mathrm{m}$, as depicted in Fig. 10(a). The capillary tubes panatrate through the drilled holes of the plate and are fastened with epoxy. One side of the plate is tightly covered with a chamber, which is pressurized in order to inject air into the water column. The water temperature is kept $23 \mathrm{C}$. The sonic speed of the water is $1491 \mathrm{~m} / \mathrm{s}$; the corresponding Tait constants are $m=7.15$ and $B=310 \mathrm{MPa}$. The void fraction, $\alpha$, ranges from 0 (no air injection) to $1 \%$. An example of the image of the injected air bubbles with $\alpha_{0}=0.0081$ is presented in Fig. 10(b). The spatial distribution of the bubbles is fairly uniform but with certain size distributions. The collapsing bubbles after the projectile impact is captured in Fig. 10(c). The shock-induced collapse is too violent to maintain their spherical shape. 
First, the evolution of a shock wave for the case of no air injection $\left(\alpha_{0}=0\right)$ is examined in Fig. 11. The strain and pressure histories at different locations along the tube direction are separately plotted in the vertical axis for clarity. Note that precursory waves (propagating essentially with the sonic speed of the tube material) precede the primary shock wave but their amplitude is too small to be visible in these plots scaled with the large amplitude of the shock. In Fig. 11(a), three different threshold levels of the hoop strain $(30 \%, 40 \%$ and $50 \%$ of the maximum strain measured at the strain gauge g1) are selected to determine the postion of the wave front; the wave speed (with its standard deviation) is then obtained from the slope of a linear least-squares fit to the wave front positions. It transpires that the wave speed is fairly constant and the dispersion resulting from the thresholding is very small. Moreover, the measured wave speed $(521 \mathrm{~m} / \mathrm{s})$ is in reasonable agreement with the Korteweg-Joukowsky velocity $(518 \mathrm{~m} / \mathrm{s}$ from $(58))$. This suggests that the linear theory works effectively for predicting the water-hammer velocity in the case without bubbles, even thougth the wave is dispersive due to structural oscillations and decays as it evolves.

Next, the case with bubbly water of $\alpha_{0}=0.0024$ is investigated in Fig. 12. As in the previous example, the propagation speed is determined from the strain histories and turns out fairly constant with small standard deviation. The wave speed $(474 \mathrm{~m} / \mathrm{s})$ is now reduced by the compressible bubbles. However, the sonic speed of the mixture (containing isothermal bubbles) is $191 \mathrm{~m} / \mathrm{s}$ from (55). The significant deviation between the measurement and the linear theory explains the nonlinear effect that results from the gas-phase compressibility. The pressure amplitude due to the shock loading is on the order of megapascals at which the bubble responce is no longer linear and the linear theory is thus incapable of properly evaluating additional compressibility associated with the compressed bubbles. It is also interesting to point out that the pressure signals are contaminated with acoustic radiation from the collapsing bubbles in the neighborhood of the pressure gauges but the fluctuations at different positions are irregular due to a random configuration of the bubbles. On the other hand, the structural response is slower and rather insensitive to such pressure fluctiations.

Finally, we make a comparison between the experiment and the FSI shock theory. To do so, we need to assign piston velocity $u H$ for the closure of the steady shock relations in Sect. 4.2. The velocity of the buffer (inserted into the top of the tube) may be considered to be representative values of $u H$. For every experimental run, the buffer position was recorded using a high-speed camera. The position history was extracted from the movie and well fitted to an exponential based on the least-squares method; the buffer velocity was determined from the fitted function. The buffer motion was found to decelerate considerably within observation periods. The details of analyzing the buffer dynamics can be found in [4]. Despite the decelerating buffer motion, the waves propagate fairly with constant speeds as presented in Figs. 


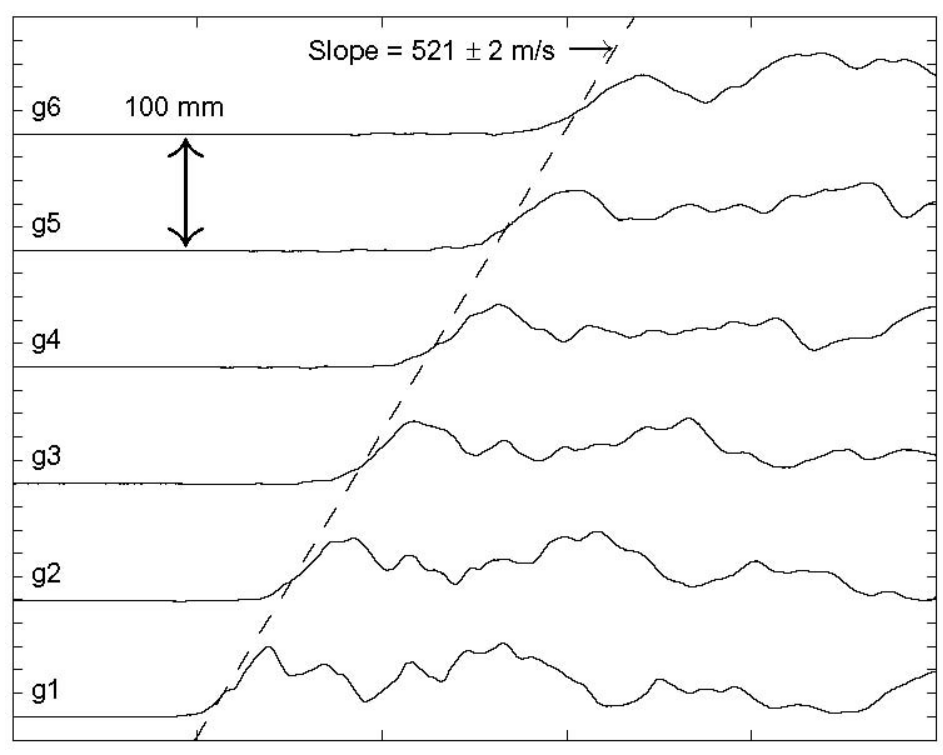

(a) 30

25

20

$10^{3} \varepsilon_{\theta}$

$012 t$ (ms) 
7.29. (a) Hoop strain. (b) Pressure.

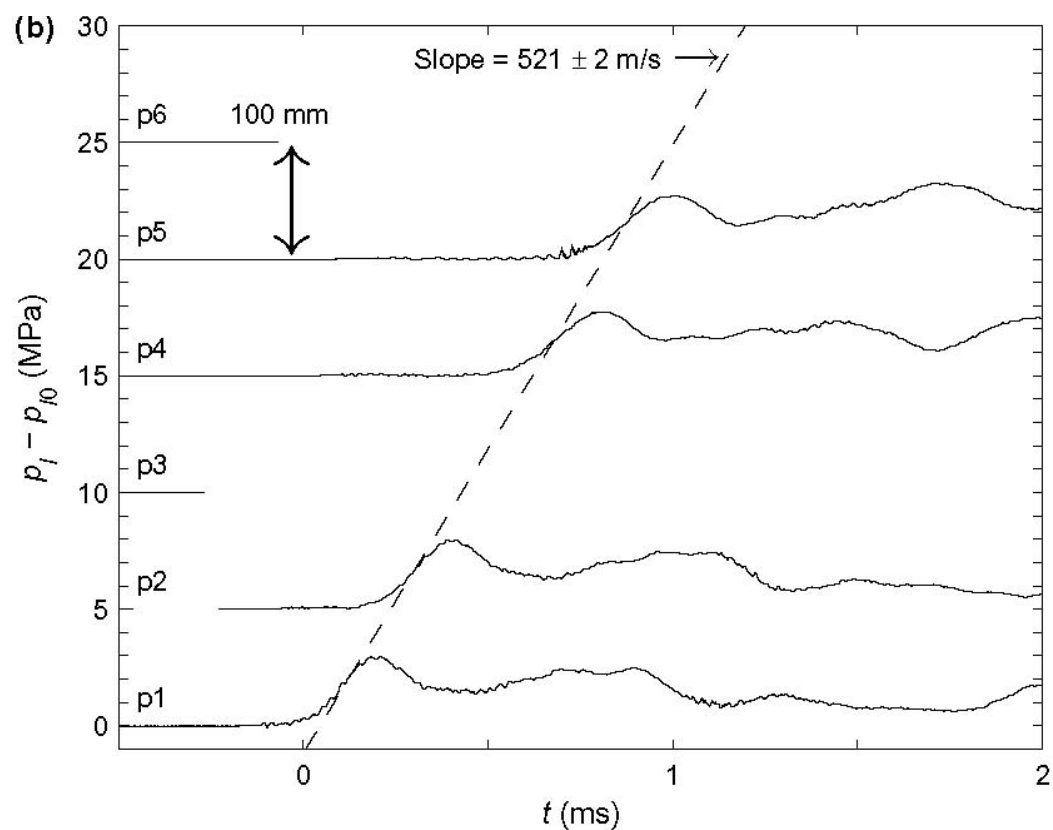

Fig. 11 An example of the evolution of shock propagation through a water-filled tube with $\xi_{l}=012 t$ (ms) 


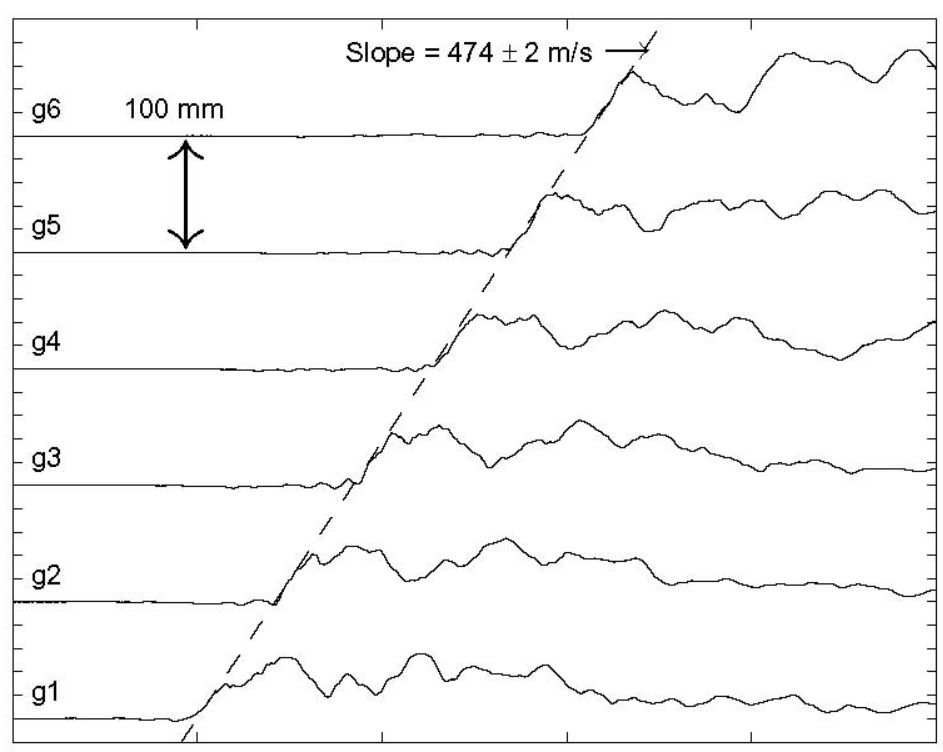

(a) 30

25

20

$10^{3} \varepsilon_{\theta}$

$012 t$ (ms) 


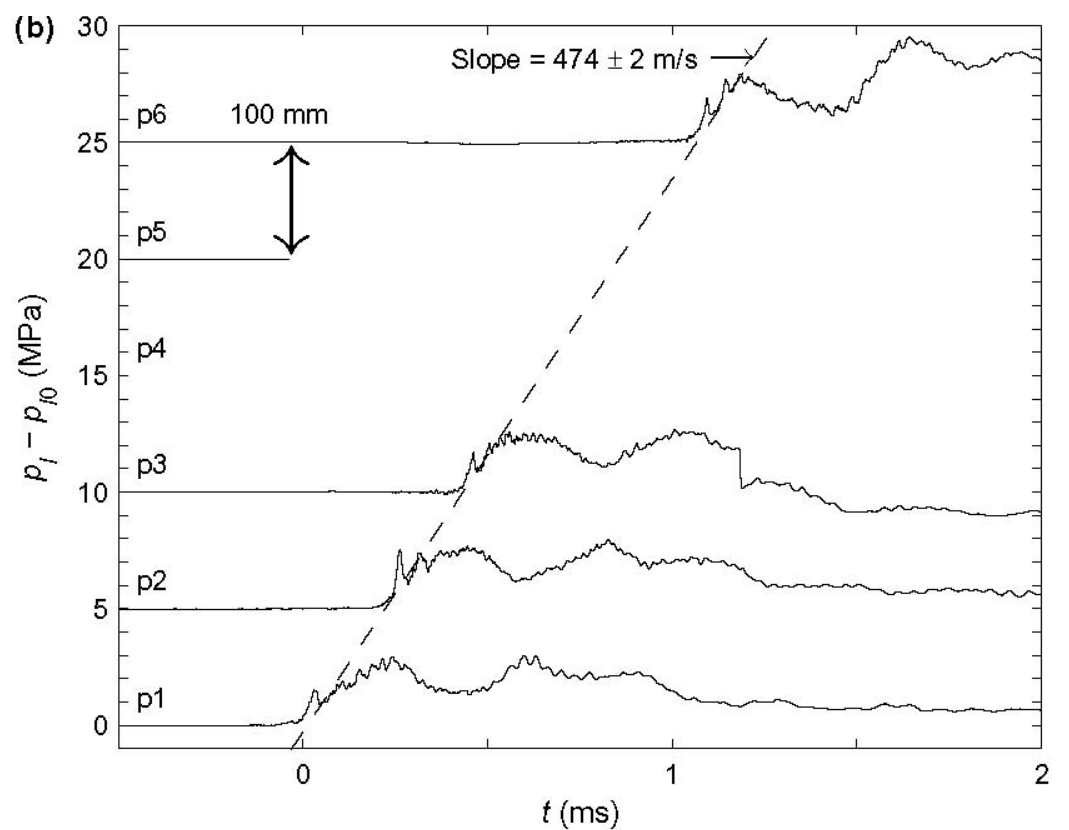

Fig. 12 An example of the evolution of shock propagation through a mixture-filled tube with $\xi_{l}=7.29$. The initial void fraction is $\alpha_{0}=0.0024$. (a) Hoop strain. (b) Pressure.

$012 t(\mathrm{~ms})$ 


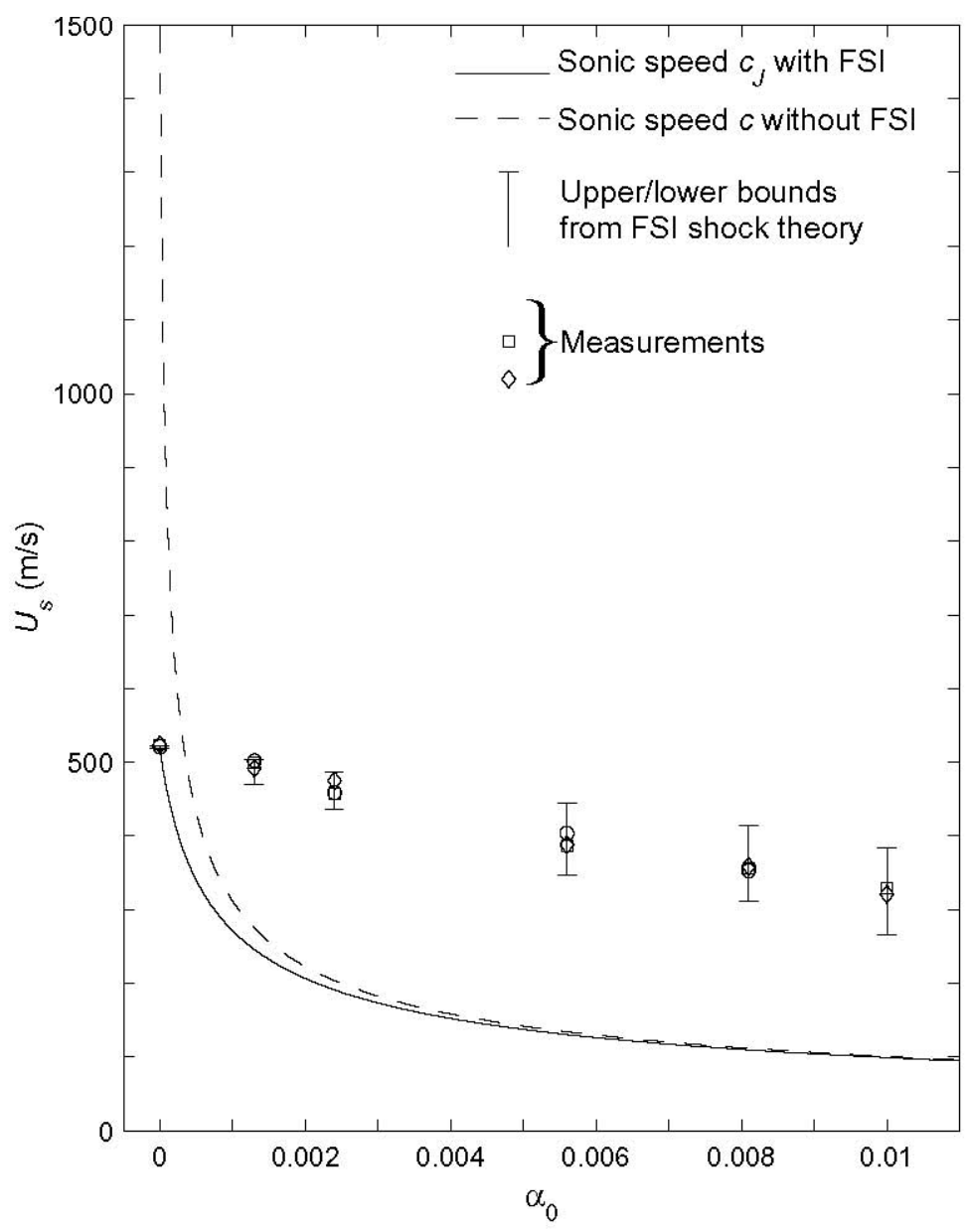

Fig. 13 Comparison of the theoretical wave speeds and the measurements from the water-hammer experiment. $0.0040 .0060 .0080 .01 \alpha 0$

11 and 12. This may be explained as follows. Initially, the shock is generated soon after the projectile collides with the buffer and starts to propagate in the fluid. At later times, the rarefaction wave arises from the deceleration of the buffer but cannot catch up the preceeding shock wave, which is propagating faster due to larger peak pressure. As a result, the peak pressure of the preceeding shock wave will not change if damping effects do not come into play (see Fig. 11(b)). Provided that the peak pressure is critical to the determination of the shock speed even in the case with bubbles, we will obtain constant shock speeds. In Fig. 13, the wave speeds from the FSI shock theory as well as the sonic speeds are plotted as a function of $\alpha_{0}$ and compared to the measured wave speeds. At each $\alpha 0$, we define the upper and lower bounds of the theoretical shock speed that correspond to the maximum and minimum piston velocities during the measurement period, respectively. Errors in the measured wave speeds and void fractions are small and omitted in this figure for clarity. It is confirmed that the choice of $и н$ is irrelevant at $\alpha_{0}=0$, for the nonlinear effect related to the liquid and structural compressibility is minor in this example. However, the measured speeds for the 
bubbly cases clearly show deviations from the sonic speeds. This explains the effect of the gas-phase nonlinearity on the propagation speeds of finite-amplitude waves. Although uncertainty associated with the choice of $u H$ is augmented as the void fraction increases, the FSI shock theory allows us to more accurately capturing the trend with increasing $\alpha_{0}$. This suggests that the gas-phase nonlinearity as well as FSI needs to be taken into account to properly estimate the propagation speeds of shock waves in a mixture-filled tube. 
In developing the FSI shock theory, we have ignored damping and wave dispersion, for simplicity, that result from the dynamics of both fluids and structures. To account for such effects, there is a need to numerically evaluate (unsteady) conservation laws for bubbly flow interacting with a deformable tube. If bubble fission occurs due to shock loading as in Fig. 10(c), the fission damping needs to be included in the modeling of single bubble dynamics $[16,29]$. Unsteady wall friction

[10] and the viscoelasticity of tube materials [76] may also affect the wave speed and damping.

\section{Concluding Remarks}

The shock dynamics of dilute bubbly flows have been reviewed. Particularly, one-dimensional shock propagation was considered as a canonical example in order to clarify fundamental issues arising in polydisperse flows in pipes. First, the conservation laws for bubbly flows with the distribution of equilibrium bubble sizes were formulated, using an ensemble-averaging technique, so as to investigate mixture-averaged dynamics. The dispersion relation for linear waves, which results from linearizing the mixture-averaged conservation equations, shows that the inclusion of broad bubble size distribution yields a profound impact on acoustic properties. Next, the propagation of weak shocks in polydisperse flows was numerically studied. A series of the numerical simulations reveal that the bubble size distribution leads to an apparent damping of the averaged shock dynamics due to phase cancelations in oscillations of the different-sized bubbles; the apparent damping with broad size distributions can dominate over physical dissipation associated with individual bubble dynamics and makes the shock profile less oscillatory. Finally, the propagation of strong shocks in a fluid-filled, deformable tube was considered. The bubbly flow equations were extended to quasi-one-dimensional configurations and the steady shock relations that account for tube deformation were derived. A comparison between the FSI shock theory and the water-hammer experiment clearly suggests that the nonlinear effect attributed to compressible bubbles (as well as FSI) needs to be taken into account to properly predict the propagation speeds of finite-amplitude waves in a mixture-filled pipe. 
Acknowledgements The authors would like to thank Toshiyuki Sanada, Kazuaki Inaba, Jason S. Damazo, and Joseph E. Shepherd for their help in the water-hammer experiments. This chapter is based mainly on the first author's PhD work [1] that was supported by the DoD MURI on Mechanics and Mechanisms of Impulse Loading, Damage and Failure of Marine Structures and Materials through the Office of Naval Research (ONR Grant No. N00014-06-1-0730).

\section{References}

1. Ando, K.: Effects of polydispersity in bubbly flows. $\mathrm{PhD}$ Thesis, California Institute of Technology (2010). Available at: http://thesis.library.caltech.edu/5859/

2. Ando, K., Colonius, T., Brennen, C.E.: Improvement of acoustic theory of ultrasonic waves in dilute bubbly liquids. J. Acoust. Soc. Am. 126, EL69-EL74 (2009)

3. Ando, K., Colonius, T., Brennen, C.E.: Numerical simulation of shock propagation in a poly-disperse bubbly liquid. Int. J. Multiphase Flow 37, 596-608 (2011)

4. Ando, K., Sanada, T., Inaba, K., Damazo, J.S., Shepherd, J.E., Colonius, T., Brennen, C.E.: Shock propagation through a bubbly liquid in a deformable tube. J. Fluid Mech. 671, 339-363 (2011)

5. Arndt, R.E.A.: Cavitation in fluid machinery and hydraulic structures. Annu. Rev. Fluid Mech. 13, 273-328 (1981)

6. Bailey, M.R., McAteer, J.A., Pishchalnikov, Y.A., Hamilton, M.F., Colonius, T.: Progress in lithotripsy research. Acoust. Today 18, 18-29 (2006)

7. Balsara, D.S., Shu, C.W.: Monotonicity preserving weighted essentially non-oscillatory schemes with increasingly high order of accuracy. J. Comput. Phys. 160, 405-452 (2000)

8. Batchelor, G.K.: The stress system in a suspension of force-free particles. J. Fluid Mech. 41 545-570 (1970)

9. Bender, C.M., Orszag, S.A.: Advanced Mathematical Methods for Scientists and Engineers. McGraw-Hill (1978)

10. Bergant, A.: Developments in unsteady pipe flow friction modeling. J. Hydraul. Res. 39, 249-257 (2001)

11. Beylich, A.E., G"ulhan, A.: On the structure of nonlinear waves in liquids with gas bubbles. Phys. Fluids A 2, 1412-1428 (1990)

12. Biesheuvel, A., van Wijngaarden, L.: Two-phase flow equations for a dilute dispersion of gas bubbles in liquid. J. Fluid Mech. 148, 301-318 (1984)

13. Bremond, N., Arora, M., Ohl, C.D., Lohse, D.: Controlled muntibubble surface cavitation. Phys. Rev. Lett. 96, 224501 (2006)

14. Brennen, C.E.: Hydrodynamics of Pumps. Oxford University Press (1994)

15. Brennen, C.E.: Cavitation and Bubble Dynamics. Oxford University Press (1995)

16. Brennen, C.E.: Fission of collapsing cavitation bubbles. J. Fluid Mech. 472, 153-166 (2002)

17. Brennen, C.E.: Fundamentals of Multiphase Flow. Cambridge University Press (2005)

18. Caflisch, R.E., Miksis, M.J., Papanicolaou, G.C., Ting, L.: Effective equations for wave prop-

agation in bubbly liquids. J. Fluid Mech. 153, 259-273 (1985)

19. Caflisch, R.E., Miksis, M.J., Papanicolaou, G.C., Ting, L.: Wave propagation in bubbly liquids at finite volume fraction. J. Fluid Mech. 160, 1-14 (1985)

20. Campbell, I.J., Pitcher, A.S.: Shock waves in a liquid containing gas bubbles. Proc. R. Soc. Lond. A 243, 534-545 (1958)

21. Carstensen, E.L., Foldy, L.L.: Propagation of sound through a liquid containing bubbles. J.

Acoust. Soc. Am. 19, 481-501 (1947)

22. Cole, R.H.: Underwater Explosions. Princeton University Press (1948)

23. Colonius, T., d'Auria, F., Brennen, C.E.: Acoustic saturation in bubbly cavitating flow adjacent to an oscillating wall. Phys. Fluids 12, 2752-2761 (2000)

24. Colonius, T., Hagmeijer, R., Ando, K., Brennen, C.E.: Statistical equilibrium of bubble oscillations in dilute bubbly flows. Phys. Fluids 20, 040902 (2008)

25. Commander, K.W., Prosperetti, A.: Linear pressure waves in bubbly liquids: Comparison between theory and experiments. J. Acoust. Soc. Am. 85, 732-746 (1989)

26. Dashpande, V.S., Heaver, A., Fleck, N.A.: An underwater shock simulator. Proc. R. Soc. A 462,

1021-1041 (2006)

27. Delale, C.F., Nas, S., Tryggvason, G.: Direct numerical simulations of shock propagation in 
bubbly liquids. Phys. Fluids 17, 121705 (2005)

28. Delale, C.F., Tryggvason, G.: Shock structure in bubbly liquids: comparison of direct numerical simulations and model equations. Shock Waves 17, 433-440 (2008)

29. Delale, C.F., Tunc, M.: A bubble fission model for collapsing cavitation bubbles. Phys. Fluids 16, 4200-4203 (2004)

30. Epstein, P.S., Plesset, M.S.: On the stability of gas bubbles in liquid-gas solutions. J. Chem. Phys. 18, 1505-1509 (1950)

31. Espinosa, H.D., Lee, S., Moldovan, N.: A novel fluid structure interaction experiment to investigate deformation of structural elements subjected to impulsive loading. Exp. Mech. 46, 805-824 (2006)

32. Feuillade, C.: The attenuation and dispersion of sound in water containing multiply interacting air bubbles. J. Acoust. Soc. Am. 99, 3412-3430 (1996)

33. Foldy, L.L.: The muntiple scattering of waves. Phys. Rev. 67, 107-119 (1945)

34. Fujikawa, S., Akamatsu, T.: Effects of the non-equilibrium condensation of vapour on the pressure wave produced by the collapse of a bubble in a liquid. J. Fluid Mech 97, 481-512 (1980)

35. Fuster, D., Colonius, T.: Modelling bubble clusters in compressible liquids. J. Fluid Mech. 688 352-389 (2011)

36. Gilmore, F.R.: The collapse and growth of a spherical bubble in a viscous compressible liquid. Hydrodynamics Laboratory Report 26-4, California Institute of Technology (1952)

37. Gottlieb, S., Shu, C.W.: Total variation diminishing Runge-Kutta schemes. Math. Compt. 67, 73-85 (1998)

38. Harten, A., Engquist, B., Osher, S., Chakravarthy, S.R.: Uniformly high order accurate essentially non-oscillatory schemes, III. J. Comput. Phys. 71, 231-303 (1987)

39. Harten, A., Lax, P.D., van Leer, B.: On upstream differencing and Godunov-type schemes for hyperbolic conservation laws. SIAM Rev. 25, 35-61 (1983)

40. Inaba, K., Shepherd, J.E.: Flexural waves in fluid-filled tubes subject to axial impact. J. Pressure

Vessel Technol. 132, 021302 (2010)

41. Ishii, M., Hibiki, T.: Thermo-Fluid Dynamics of Two-Phase Flow. Springer (2006)

42. Johnsen, E., Colonius, T.: Numerical simulations of non-spherical bubble collapse. J. Fluid Mech. 629, 231-262 (2009)

43. Joukowsky, N.E.: Memoirs of the Imperial Academy Society of St. Petersburg. Proc. Amer. Water Works Assoc. 24, 341-424 (1898)

44. Kameda, M., Matsumoto, Y.: Shock waves in a liquid containing small gas bubbles. Phys. Fluids 8, 322-335 (1996)

45. Kameda, M., Shimaura, N., Higashino, F., Matsumoto, Y.: Shock waves in a uniform bubbly flow. Phys. Fluids 10, 2661-2668 (1998)

46. Kedrinskii, V.K.: Hydrodynamics of Explosion. Springer (2005)

47. Kobori, T., Yokoyama, S., Miyashiro, H.: Propagation velocity of pressure wave in pipe line. Hitachi Hyoron 37, 33-37 (1955)

48. Korteweg, D.J.: Ueber die Fortpflanzungsgeschwindigkeit des Shalles in elastischen R"ohren. Annalen der Physik und Chemie 5, 525-542 (1878)

49. Krimmel, J., Colonius, T., Tanguay, M.: Simulation of the effects of cavitation and and anatomy in the shock path of model lithotripters. Urol. Res. 38, 505-518 (2010)

50. LeVeque, R.J.: Numerical Methods for Conservation Laws. Birkh"auser Verlag (1992)

51. LeVeque, R.J.: Finite Volume Methods for Hyperbolic Problems, second edn. Cambridge University Press (2002)

52. Liu, X.D., Osher, S., Chan, T.: Weighted essentially non-oscillatory schemes. J. Comput. Phys.

115, 200-212 (1994)

53. Lu, T., Samulyak, R., Glimm, J.: Direct numerical simulation of bubbly flows and application to cavitation mitigation. J. Fluids Eng. 129, 595-604 (2007)

54. Minnaert, M.: On musical air-bubbles and sounds of running water. Phil. Mag. 16, 235-248 (1933)

55. Nagayama, K., Mori, Y., Shimada, K.: Shock Hugoniot compression curve for water up to 1 GPa by using a compressed gas gun. J. Appl. Phys. 91, 476-482 (2002)

56. Nigmatulin, R.I.: Spatial averaging in the mechanics of heterogeneous and dispersed systems. Int J. Heat Mass Transfer 5, 353-385 (1979)

57. Nigmatulin, R.I.: Mathematical modelling of bubbly liquid motion and hydrodynamical effects in wave propagation phenomenon. Appl. Sci. Res. 38, 267-289 (1982)

58. Nigmatulin, R.I., Khabeev, N.S., Hai, Z.N.: Waves in liquids with vapour bubbles. J. Fluid Mech. 
186, 85-117 (1988)

59. Nigmatulin, R.I., Khabeev, N.S., Nagiev, F.B.: Dynamics, heat and mass transfer of vapour-gas bubbles in a liquid. Int. J. Heat Mass Transfer 24, 1033-1044 (1981)

60. Noordzij, L., van Wijngaarden, L.: Relaxation effects, caused by relative motion, on shock waves in gas-bubble/liquid mixtures. J. Fluid Mech. 66, 115-143 (1974)

61. Plesset, M.S.: The dynamics of cavitation bubbles. J. Appl. Mech. 16, 228-231 (1949)

62. Plesset, M.S., Prosperetti, A.: Bubble dynamics and cavitation. Annu. Rev. Fluid Mech. 9,

145-185 (1977)

63. Preston, A.T., Colonius, T., Brennen, C.E.: A reduced-order model of diffusive effects on the dynamics of bubbles. Phys. Fluids 19, 123302 (2007)

64. Prosperetti, A.: Thermal effects and damping mechanisms in the forced radial oscillations of gas bubbles in liquids. J. Acoust. Soc. Am. 61, 17-27 (1977)

65. Prosperetti, A.: Fundamental acoustic properties of bubbly liquids. In: M. Levy, H.E. Bass,

R.R. Stern (eds.) Handbook of Elastic Properties of Solids, Liquids, and Gases (Volume 4. Elastic

Properties of Fluids: Liquids and Gases), pp. 183-205. Academic (2001)

66. Prosperetti, A., Crum, L.A., Commander, K.W.: Nonlinear bubble dynamics. J. Acoust. Soc. Am. 83, 502-514 (1988)

67. Qiu, J., Shu, C.W.: On the construction, comparison, and local characteristic decomposition for high-order central WENO schemes. J. Comput. Phys. 183, 187-209 (2002)

68. Ranjan, D., Oakley, J., Bonazza, R.: Shock-bubble interactions. Annu. Rev. Fluid Mech. 43, 117-140 (2011)

69. Rayleigh, L.: On the pressure developed in a liquid during the collapse of a spherical cavity. Phil. Mag. 34, 94-98 (1917)

70. Seo, J.H., Lele, S.K., Tryggvason, G.: Investigation and modeling of bubble-bubble interaction effect in homogeneous bubbly flows. Phys. Fluids 22, 063302 (2010)

71. Shepherd, J.E., Inaba, K.: Shock loading and failure of fluid-filled tubular structures. In:

A. Shukla, G. Ravichandran, Y.D.S. Rajapakse (eds.) Dynamic Failure of Materials and Structures, pp. 153-190. Springer (2010)

72. Shu, C.W.: Essentially non-oscillatory and weighted essentially non-oscillatory schemes for hyperbolic conservation laws. ICASE Report 97-65, NASA Langley Research Center (1997)

73. Shu, C.W., Osher, S.: Efficient implementation of essentially non-oscillatory shock-capturing schemes. J. Comput. Phys. 77, 439-471 (1988)

74. Skalak, R.: An extension of the theory of water hammer. Trans. ASME 78, 105-116 (1956) 75. Smereka, P.: A Vlasov equation for pressure wave propagation in bubbly fluids. J. Fluid Mech. 454, 287-325 (2002)

76. Suo, L., Wylie, E.B.: Complex wavespeed and hydraulic transients in viscoelastic pipes. J. Fluids Eng. 112, 496-500 (1990)

77. Takahira, H.: A remark on the pressure terms in the Rayleigh-Plesset equation for cavitating flows. Trans. Jpn. Soc. Mech. Eng. B 70, 617-622 (2004)

78. Tan, M.J., Bankoff, S.G.: Propagation of pressure waves in bubbly mixtures. Phys. Fluids 27, 1362-1369 (1984)

79. Tan, M.J., Bankoff, S.G.: Strong shock waves propagating through a bubbly mixture. Exp. Fluids 2, 159-165 (1984)

80. Tanguay, M.: Computation of bubbly cavitating flow in shock wave lithotripsy. PhD Thesis,

California Institute of Technology (2004). Available at: http://thesis.library.caltech.edu/2188/

81. Thompson, P.A.: Compressible-Fluid Dynamics. McGraw-Hill (1972)

82. Tijsseling, A.S., Lambert, M.F., Simpson, A.R., Stephens, M.L., V'ttkovsk'y, J.P., Bergant, A.:

Skalak's extended theory of water hammer. J. Sound Vib. 310, 718-728 (2008)

83. Toro, E.F.: Riemann Solvers and Numerical Methods for Fluid Dynamics: A Practical Introduction, third edn. Springer (2009)

84. Toro, E.F., Spruce, M., Speares, W.: Restoration of the contact surface in the HLL-Riemann solver. Shock Waves 4, 25-34 (1994)

85. van Wijngaarden, L.: On the equations of motion for mixtures of liquid and gas bubbles. J. Fluid Mech. 33, 465-474 (1968)

86. van Wijngaarden, L.: One-dimensional flow of liquids containing small gas bubbles. Annu. Rev. Fluid Mech. 4, 369-396 (1972)

87. Watanabe, M., Prosperetti, A.: Shock waves in dilute bubbly liquids. J. Fluid Mech. 274, 349-381 (1994)

88. Waterman, P.C., Truell, R.: Multiple scattering of waves. J. Math. Phys. 2, 512-537 (1961) 
89. Wylie, E.B., Streeter, V.L.: Fluid Transients in Systems. Prentice Hall (1993)

90. Zhang, Z.D., Prosperetti, A.: Averaged equations for inviscid disperse two-phase flow. J. Fluid

Mech. 267, 185-219 (1994)

91. Zhang, Z.D., Prosperetti, A.: Ensemble-averaged equations for bubbly flows. Phys. Fluids 6,

2956-2970 (1994)

92. Zhang, Z.D., Prosperetti, A.: Momentum and energy equations for disperse two-phase flows and

their closure for dilute suspensions. Int. J. Multiphase Flow 23, 425-453 (1997) 